\title{
Electrospun nanofibrous materials for wound healing applications
}

\author{
B. Balusamy, A. Senthamizhan, T. Uyar \\ Bilkent University, Ankara, Turkey
}

\subsection{Introduction}

Developments in regenerative medicine and tissue engineering over the decades advanced wound healing methods for enhancing healing process and also overcome existing complications. Archetypically, wound is defined as a disruption of cellular community that consequences in separation of normally connected tissues or a type of injury resulted from the skin that is torn, cut, or punctured [1]. The breach occurred by skin damage leads to bacterial infection that causes inflammation and infection locally or systemically. The wound healing is not a simple linear process, which is one of the most complex and dynamic processes that involved with multiple biological pathways, blood elements, cells, growth factors, and extracellular matrix (ECM) [2-4].

Eventually, wound healing occurs through three stages, namely, (a) inflammation, (b) new tissue formation, and (c) remodeling. The inflammation stage of wound healing process happens immediately following the tissue damage and lasts about $48 \mathrm{~h}$. During this stage, multiple biological pathways are initiated to prevent blood and fluid losses and infection and remove dead and devitalized (dying) tissues. The second stage of the wound healing process occurs between 2 and 10 days after injury and which is characterized by cellular proliferation and migration of different cell types. The third stage of the wound healing is remodeling, and it begins after $2-3$ years following the injury and lasts for a year or more in which all the process activated after injury wind down and cease. The detailed information on different stages of the wound repair process is depicted in Fig. 8.1 [4]. Based on the wound repair process, wounds are classified into acute and chronic wounds. Acute wounds are generally healing completely within 8-12 weeks time frame, whereas the chronic wounds take more than 12 weeks for healing process $[5,6]$. Wound management complications are always having significant impacts in clinical burden; thus, the wound dressing market has great economic potential worldwide [7]. Therefore, recent decades witnessed that a variety of wound dressing materials resulted from numerous approaches have been introduced at different aspects of wound healing process [8].

Development of wound dressing can be tracked back since ancient era from application of medicinal plant, animal fat, and honey to tissue engineering scaffolds. An 


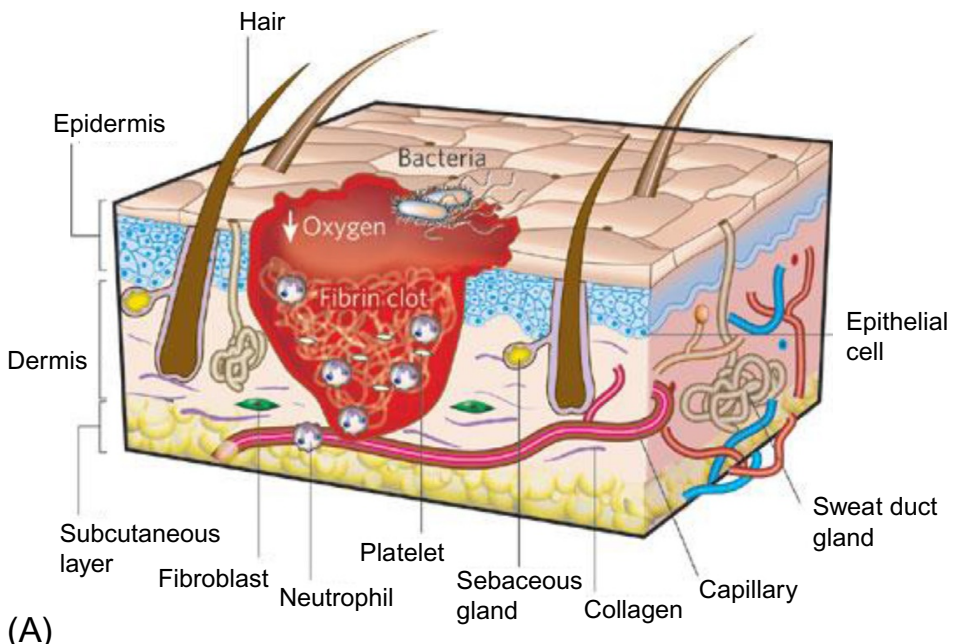

(A) gland Collagen Capillary

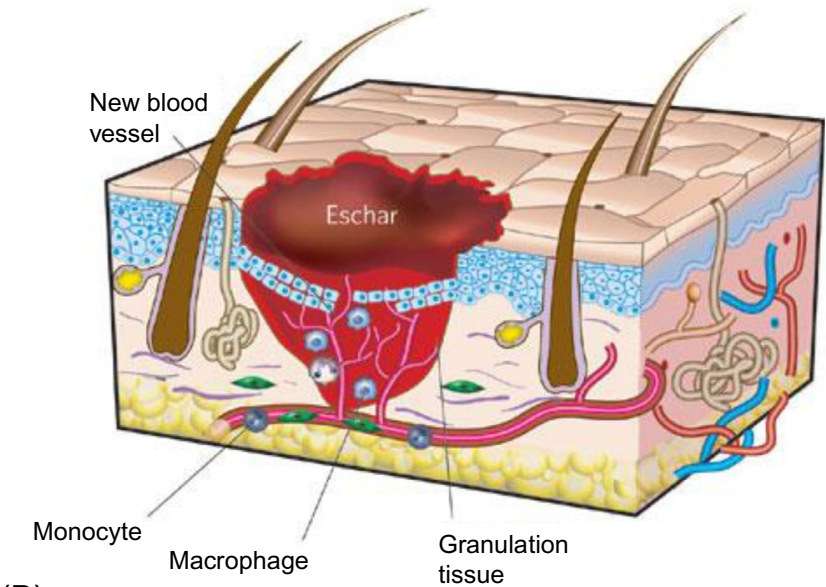

(B) tissue

(C)

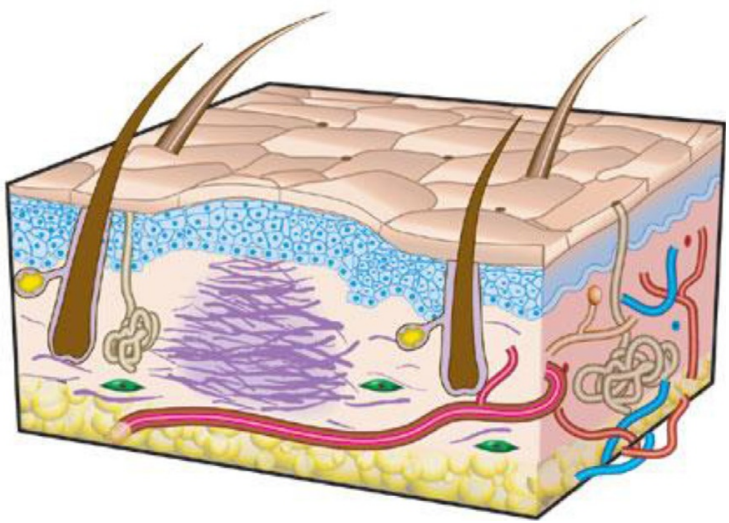

Fig. 8.1 See legend on opposite page. 
ideal wound dressing is expected to possess the characteristics including provide or maintain a moist wound environment, removal of blood and excess exudates, gaseous exchange, protect the wound from microorganism invasion, protect the wound from trauma, thermal insulation provision, and most importantly cost-effective and less frequency of dressing change [8,9]. Clinical practice has been widely adopted plain gauze for wound care owing to their inexpensive and readily available nature, but still, it has many shortcomings that has inspired to develop variety of other materials including foams, adhesive films, hydrogels, alginates, hydrocolloids, and biological dressings possessing remarkable features $[8,10]$. In the recent past, nanotechnology-based therapy has significantly revolutionized the management of wound care and also recognized as possible next-generation therapy. To date, a wide range of nanomaterials composed of carbon, lipid, ceramic, metal, and metal oxide nanoparticles have been reported for their beneficial role in wound healing [11].

Electrospun nanofibers have received tremendous attention toward energy, environmental, and biomedical applications due to their exceptional characteristic features [12-16]. Electrospun nanofiber scaffolds offer ideal characteristics of wound dressings, that is, high surface area to volume ratio, adsorption of exudates, interconnected nanoporosity, controlled drug or biomolecules release, cell respiration, flexibility, and better sorption of proteins; thus, the electrospun nanofibers produced from different electrospinning approaches including blend, coaxial, emulsion electrospinning, and also postspinning modifications have been extensively used in wound healing applications [17-22]. Different biomolecules involved wound healing, fabrication of nanofibers, and current strategies used in electrospinning to produce wound dressing material were reviewed by Abrigo et al. [17]. Furthermore, the architectural feature of electrospun nanofibers mimics the ECM structure, which is the most important characteristic of any scaffold intended to use in tissue engineering applications since it influences the cell binding. Stevens and George [23] reported that the scaffolds with

Fig. 8.1 Classic stages of wound repair. There are three classic stages of wound repair: (A) inflammation, (B) new tissue formation, and (C) remodeling. (A) Inflammation. This stage lasts until about $48 \mathrm{~h}$ after injury. Depicted is a skin wound at about $24-48 \mathrm{~h}$ after injury. The wound is characterized by a hypoxic (ischaemic) environment in which a fibrin clot has formed. Bacteria, neutrophils, and platelets are abundant in the wound. Normal skin appendages (such as hair follicles and sweat duct glands) are still present in the skin outside the wound. (B) New tissue formation. This stage occurs about 2-10 days after injury. Depicted is a skin wound at about 5-10 days after injury. An eschar (scab) has formed on the surface of the wound. Most cells from the previous stage of repair have migrated from the wound, and new blood vessels now populate the area. The migration of epithelial cells can be observed under the eschar.

(C) Remodeling. This stage lasts for a year or longer. Depicted is a skin wound about 1-12 months after repair. Disorganized collagen has been laid down by fibroblasts that have migrated into the wound. The wound has contracted near its surface, and the widest portion is now the deepest. The reepithelialized wound is slightly higher than the surrounding surface, and the healed region does not contain normal skin appendages.

Reproduced with permission from Gurtner GC, Werner S, Barrandon Y, Longaker MT. Wound repair and regeneration. Nature 2008;453:314-21 with permission from Nature Publishing Group. 
nanoscale architecture possess more binding sites due to higher surface area than the microscale architecture scaffold.

This chapter highlights wide range of polymeric electrospun nanofibers and incorporated active agents intended for wound healing applications rather than discussing various adopted electrospinning approaches for preparing nanofibrous wound dressing materials. A well-demonstrated example of such scaffolds has been discussed briefly under each section. In addition, current challenges associated with development of wound care materials and electrospun nanofibrous scaffolds in the market for wound healing are also presented.

\subsection{Electrospun nanofibrous scaffolds in wound healing}

Electrospun nanofibrous scaffolds in wound healing applications have been well demonstrated using a variety of natural, synthetic, and composite polymers and functionalization of fibers with active agents and herbal molecules [24-26]. A well-demonstrated example of each material is discussed under respective section of the chapter.

\subsubsection{Natural polymer nanofibers as wound healing scaffolds}

The electrospun nanofibrous scaffolds prepared using natural polymers possess excellent biodegradability, biocompatibility, and other biological properties recognized by cells for physiological process. To date, numerous nanofibrous scaffolds prepared from variety of natural polymers demonstrated their potency in enhanced wound healing including collagen, gelatin, hyaluronic acid, chitosan, and silk fibroin [27]. Collagen is an insoluble fibrous protein, is abundant in the animal kingdom, and is the main component of ECM, providing tensile strength and structural integrity to tissues. Powell et al. [28] prepared freeze-dried (FD) and electrospun (ES) collagen scaffolds further populated with human dermal fibroblasts (HF) and epidermal keratinocytes (HK), which have been isolated from surgical discard tissue to prepare freeze-dried collagen skin substitutes (FCSS) and electrospun collagen skin substitutes (ECSS), and then studied their potency in wound healing. In vitro studies indicated that both FCSS and ECSS did not reveal any significant differences in cell proliferation, surface hydration, or cellular organization and exhibited excellent stratification with a continuous layer of basal keratinocytes present at the dermalepidermal junction. Immunostaining for involucrin showed positive staining in all the epidermal layers except for the basal cell layer in both the FDSS and ECSS as shown in Fig. 8.2. Basement membrane formation was evident in both the FDSS and ECSS as indicated by the continuous layer of collagen IV at the dermal-epidermal junction in the skin substitutes in vitro.

The wound healing study in athymic mice demonstrated that prepared grafts prepared with FD and ES scaffolds were well integrated into surrounding murine skin and possessed a uniformly dry epidermis (Fig. 8.3A and B). The FCSS and ECSS grafts were visibly smaller with a majority of the ECSS maintaining their original shape as shown in Fig. 8.3C and D upon 8 weeks exposure, but differences in surface area 

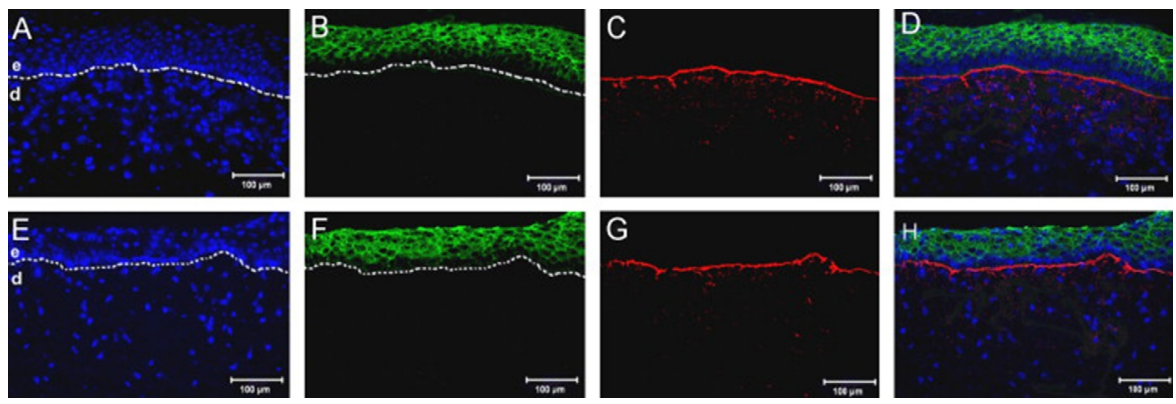

Fig. 8.2 Immunohistological images of FCSS (A-D) and ECSS (E-H) showing cell nuclei (A and $\mathrm{E}$ ), human involucrin (B and F), human collagen type IV (C and $\mathrm{G})$, and merged images (D and $\mathrm{H})$. The epidermis and dermis are denoted by e and d, respectively. The dashed line indicates the dermal-epidermal junction. Scale bar $=100 \mu \mathrm{m}$.

Reproduced with permission from Powell HM, Supp DM, Boyce ST. Influence of electrospun collagen on wound contraction of engineered skin substitutes. Biomaterials 2008;29:834-43 with permission from Elsevier.
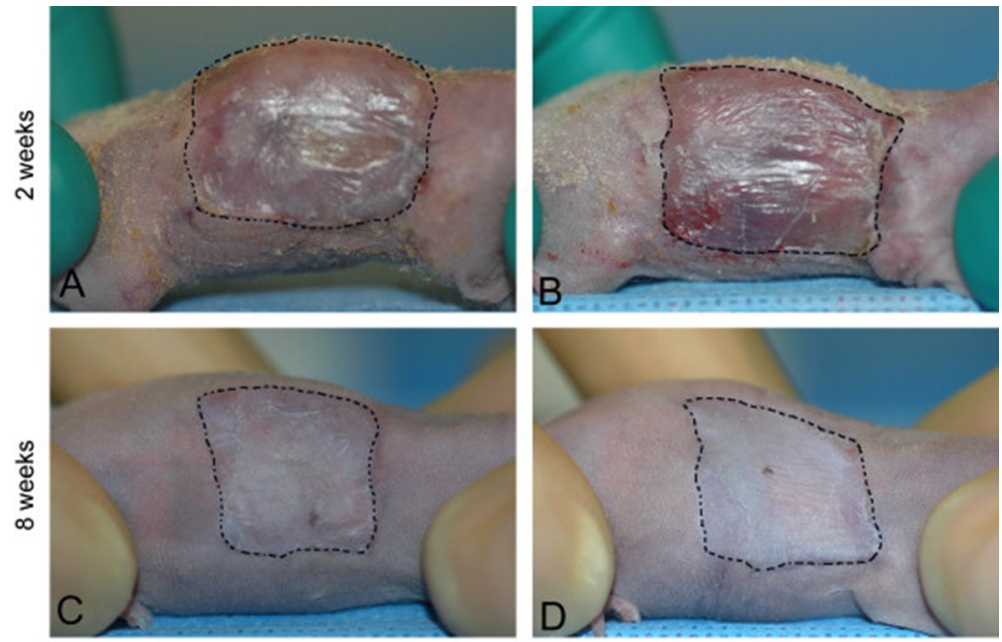

FCSS

ECSS

Fig. 8.3 Appearance of grafts on athymic mice 2 and 8 weeks after grafting. Skin substitutes fabricated using freeze-dried (FCSS; A and C) or electrospun collagen (ECSS; B and D) at 2 weeks (A and B) or 8 weeks (C and D), respectively. Wound areas are traced with a dashed black line.

Reproduced with permission from Powell HM, Supp DM, Boyce ST. Influence of electrospun collagen on wound contraction of engineered skin substitutes. Biomaterials 2008;29:834-43 with permission from Elsevier. 
between the FCSS and ECSS groups were observed with the ECSS grafts on average larger than the FCSS. Further, the microscopic evaluation of skin biopsy collected from each animal showed excellent cell migration into the dermal component of both graft types. However, undigested bovine collagen sponge was visible in within the graft on animals from the FCSS group, whereas no residual ES collagen was found in any of the ECSS animals at week 8 and the dermal component of the grafted ECSS and the murine skin. And also, it was found that after grafting to full-thickness wounds in athymic mice, both skin substitutes had high rates of engraftment, $87.5 \%$ in the FCSS group and $100 \%$ in the ECSS group.

Similarly, in a recent study, tilapia skin collagen electrospun nanofibers have showed rapid and effective wound healing property [29]. The wound healing efficiency of tilapia collagen nanofibers was investigated using Sprague-Dawley (SD) rat models with dorsal full-thickness skin defects. The wound healing study results demonstrated that the significant rate of healing in animal treated with tilapia collagen nanofibers started to disappear at day 7 , and most of the wound area were covered with a continuous epidermis at day 14 as compared with untreated control and the animals treated with Kaltostat, a commonly used wound dressing. Further, the histopathologic results confirmed that the collagen nanofibers caused lowest degree of inflammatory response and induced the best growth status of new epidermis throughout wound healing process (Fig. 8.4). The inflammatory response was significantly reduced at day 7 , and new epidermis with intact structure and good continuity could also been found at day 14 . The epidermal cells were fully differentiated, basal cells were closely arranged, the horny layer could be observed, and layers of keratinocytes were evident.

Gelatin (GE) is a derivative of native collagen obtained by partial hydrolysis and has lesser immunogenicity than collagen. The wound healing applications of pure GE nanofibers are limited due to complication in mechanical properties and degradation profile. Powell and Boyce [30] fabricated porous nonwoven fibrous GE scaffolds using electrospinning technique with various solution concentrations and further investigated their influence in dermal and epidermal tissue regeneration. The study results revealed that the nanofibrous scaffolds with interfiber distance of $>5 \mu \mathrm{m}$ offered deeper penetration of $\mathrm{HF}$ and also interfiber distances $\leq 10 \mu \mathrm{m}$ exhibited well-stratified dermal and epidermal layers. Therefore, the GE nanofibrous scaffolds with 5-10 $\mu \mathrm{m}$ favored the formation of skin substitutes in vitro. Another study demonstrated the preparation of GE and PCL nanofibers using needleless electrospinning technique and further characterized their biocompatibility and therapeutic efficiency using in vitro cell cultures and in an experimental rat model with wound [31]. Cell proliferations of human mesenchymal stem cells (hMSCs), HF, and keratinocytes on the nanofibers were examined by staining the actin cytoskeleton with phalloidin and visualized by fluorescent microscopy. The in vitro study outcome indicated that significantly higher cell proliferation was observed on GE rather than the PCL nanofibers. Likewise, significantly faster wound closure was found for GE nanofibers on days 5 and 10 compared with the control group treated with gauze as shown in Fig. 8.5. No significant differences in wound closure were observed between the PCL-treated and control groups. Further, the presence of myofibroblasts was assessed at day 10 by quantifying the $\alpha$-smooth muscle actin (SMA) immunofluorescent staining (Fig. 8.6). The myofibroblast distribution in the wound area corresponded to the degree 


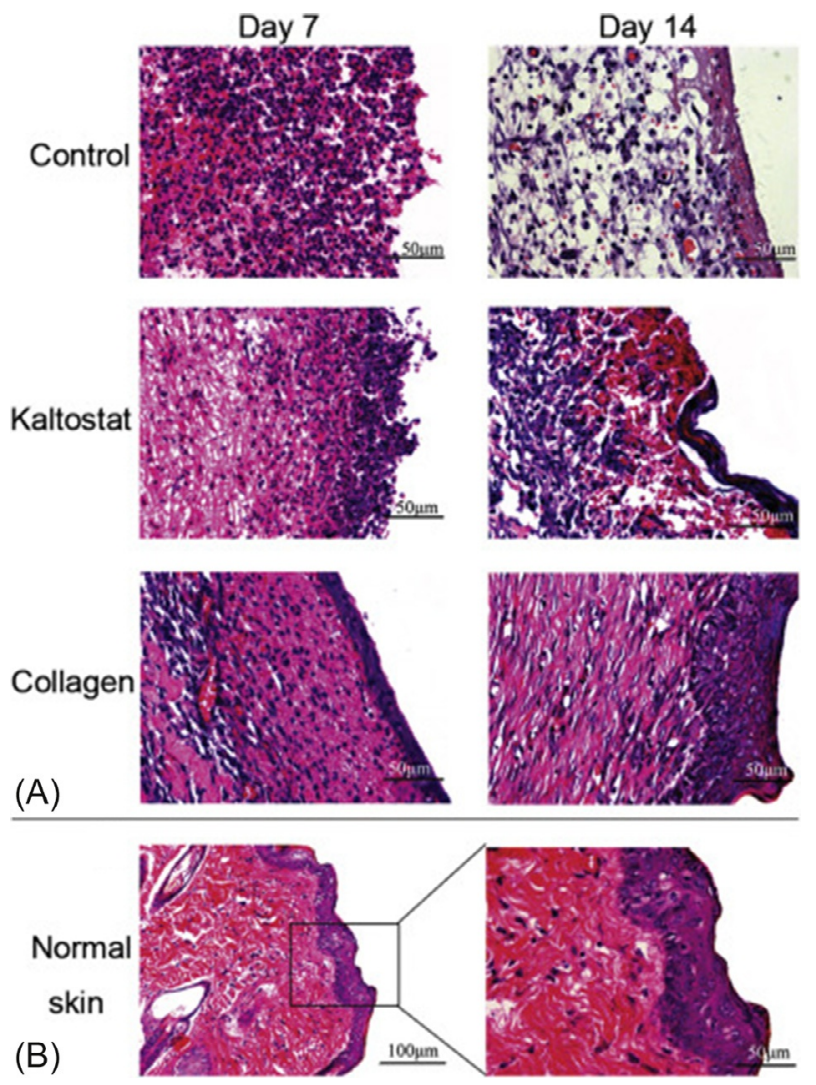

Fig. 8.4 Representative images of hematoxylin and eosin (H and E) staining. (A) Wound sections treated with tilapia collagen nanofibers or Kaltostat, with untreated wounds as control group, at days 7 and 14. (B) Normal skin.

Reproduced with permission from Zhou T, Wang N, Xue Y, Ding T, Liu X, Mo X, et al. Electrospun tilapia collagen nanofibers accelerating wound healing via inducing keratinocytes proliferation and differentiation. Colloids Surf B 2016;143:415-22 with permission from Elsevier.

of wound contraction at day 10 , and the results demonstrated significantly higher area fraction of $\alpha$-SMA staining, which was found for GE and which clearly indicates that GE-treated group wounds were more contracted than other groups.

Hyaluronic acid (HA) is a naturally occurring polysaccharide and one of the chief components of ECM. The HA is proved to be beneficial in wound healing applications since it plays major role in facilitating keratinocyte migration and proliferation, activating and moderating the inflammatory response, and reducing scar formation. A preclinical study was conducted to compare healing of wounds covered by an adhesive bandage, a sterilized solid HA, a gauze with Vaseline, an antibiotic dressing, and a sterilized HA nanofiber wound dressing on crossbred pigs as an experimental model [32]. The study results showed that the sterilized HA nanofiber wound dressing exhibited better 


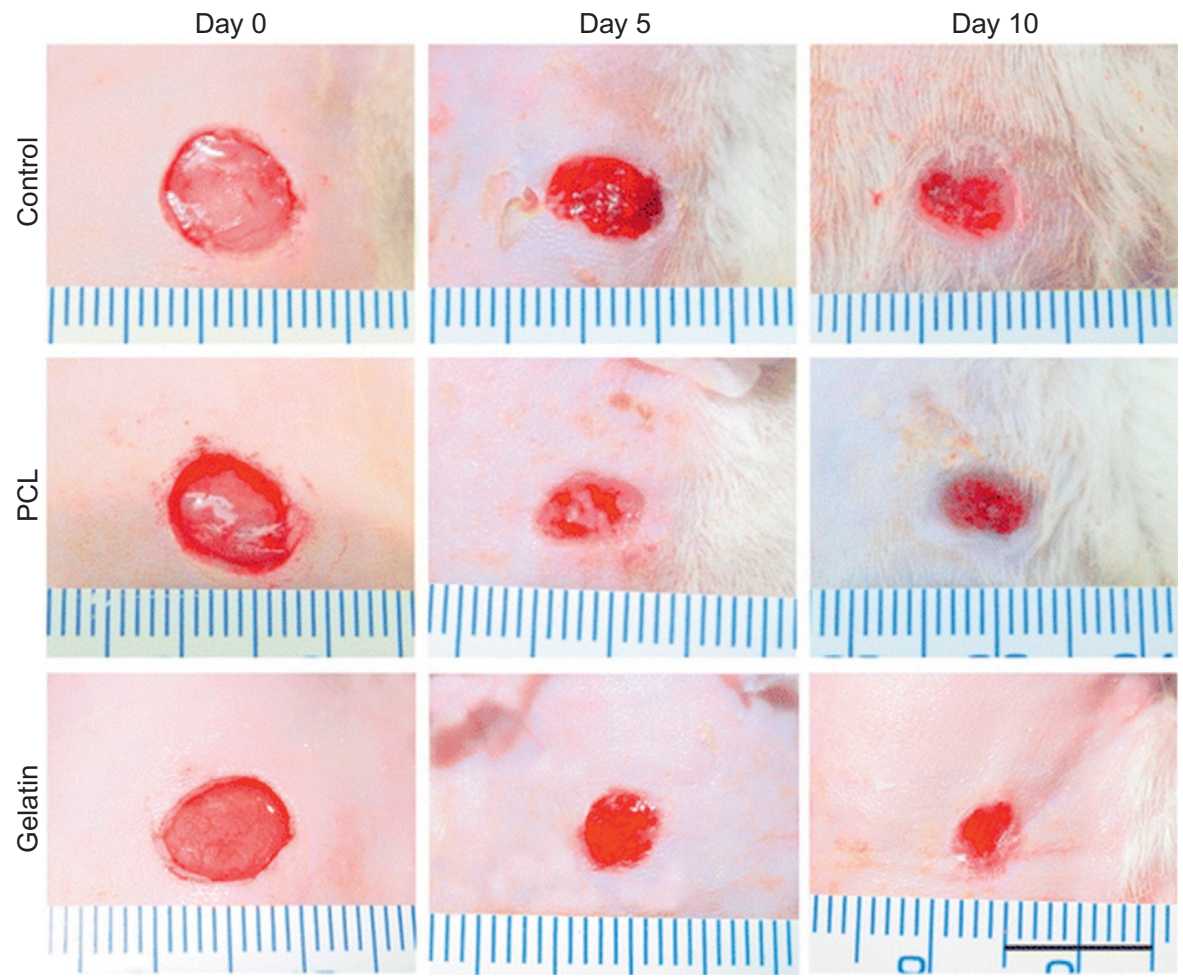

Fig. 8.5 Photographs of full-thickness skin wounds and subsequent wound contraction on days 5 and 10 after treatment with gauze (control), PCL, and gelatin nanofibers. Scale bar $=1 \mathrm{~cm}$. Reproduced with permission from Dubský M, Kubinová Š, Širc J, Voska L, Zajícěk R, Zajícová A, et al. Nanofibers prepared by needleless electrospinning technology as scaffolds for wound healing. J Mater Sci Mater Med 2012;23:931-41 with permission from Springer.

healing efficiency than adhesive bandage, sterilized solid HA, and gauze with Vaseline dressing, but it was not statistically better than the antibiotic dressing. It was reported that the enhanced wound healing performance is due to HA aids in cell migration, cell proliferation, angiogenesis, and phagocytosis, and also nanofibers can pick up the exudates from the wound to a greater extent. Another study by Ji et al. [33] reported the preparation of a cross-linked HA hydrogel nanofibers by a reactive electrospinning method. A thiolated HA derivative, 3,3'-dithiobis(propanoic dihydrazide)-modified HA (HA-DTPH), and poly(ethylene glycol) diacrylate (PEGDA) were adopted as cross-linking agents, and the cross-linking reaction was occurred simultaneously during the electrospinning process. A cell morphology study on fibronectin (FN)-adsorbed HA nanofibrous scaffolds showed that the migration of NIH 3 T3 fibroblasts into the scaffold through the nanofibrous network occurred, and a three-dimensional dendritic morphology has been demonstrated. Since the high molecular weight and hygroscopic nature of the HA lead to complications in successful preparation of nanofibers, the HA is often blended with other polymers for electrospinning process. 

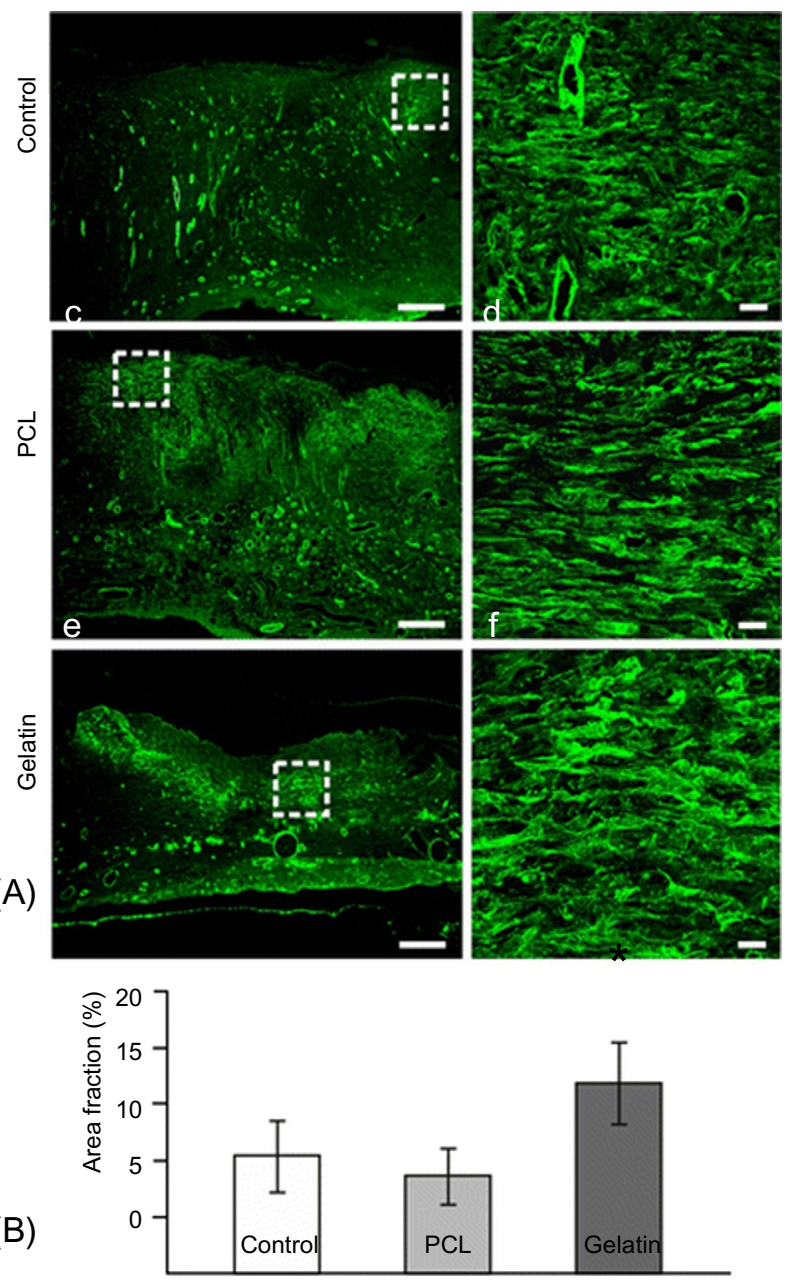

Fig. 8.6 (A) Representative micrographs of $\alpha$-SMA staining for myofibroblasts and blood vessels in wounds 10 days after treatment with control (a and b), PCL (c and d), and gelatin (e and $\mathrm{f}$ ). The right micrographs $(\mathrm{b}, \mathrm{d}$, and $\mathrm{f}$ ) represent a higher magnification view of the marked areas in the left micrographs. Scale bar, $200 \mu \mathrm{m}$ (a, c, and e) and $20 \mu \mathrm{m}$ (b, d, and f). (B) Quantification of $\alpha$-SMA immunostaining on day 10 after control treatment, PCL, and gelatin nanofibers. $\alpha$-SMA expression was significantly increased for gelatin compared with control treatment. Mann-Whitney test, ${ }^{*} P<.05,{ }^{* *} P<.01$.

Reproduced with permission from Dubský M, Kubinová Š, Širc J, Voska L, Zajícěk R, Zajícová A, et al. Nanofibers prepared by needleless electrospinning technology as scaffolds for wound healing. J Mater Sci Mater Med 2012;23:931-41 with permission from Springer. 
Chitosan, a second most abundant polysaccharide in nature and is a derivative of deacetylated chitin. The chitosan has demonstrated to have great role in wound and burn treatments owing to their hemostatic, antifungal, antibacterial, and wound healing properties. Tchemtchoua et al. [34] compared the biological property of chitosan nanofibrous scaffolds with chitosan films and FD sponges. An initial cell adhesion property of chitosan nanofibers with human fibroblasts, microvascular endothelial cells, and keratinocytes resulted in attachment of cells on day 1 and followed by well spread of cells at day 3 (Fig. 8.7). Further, it has been noted that the keratinocytes formed clusters of flat cells by tightly joining together at day 7, which is a physiological and essential property of keratinocytes in vivo for forming a physical tight barrier.

The electrospun chitosan nanofibrous scaffold also demonstrated good biocompatibility than the FD sponges during implantation study. Further, the wound healing study on mice with full-thickness wounds ( $8 \mathrm{~mm}$ in diameter) in the back skin showed that the chitosan nanofibrous scaffolds were found to adhere uniformly to freshly

Day 1

Day 3

Day 7

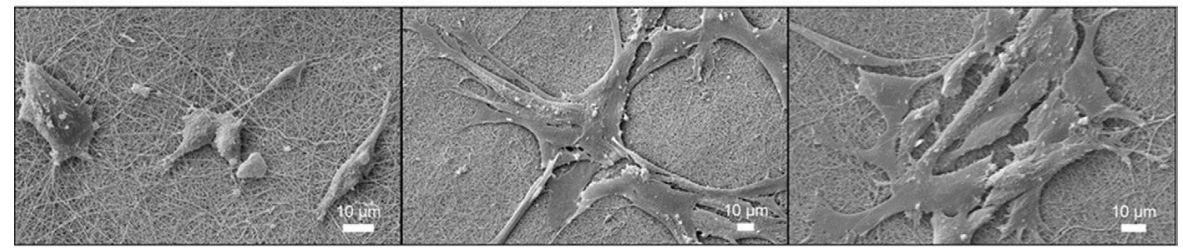

(A)

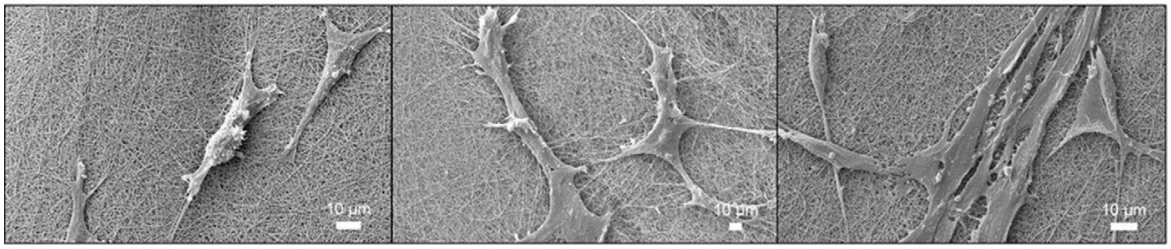

(B)

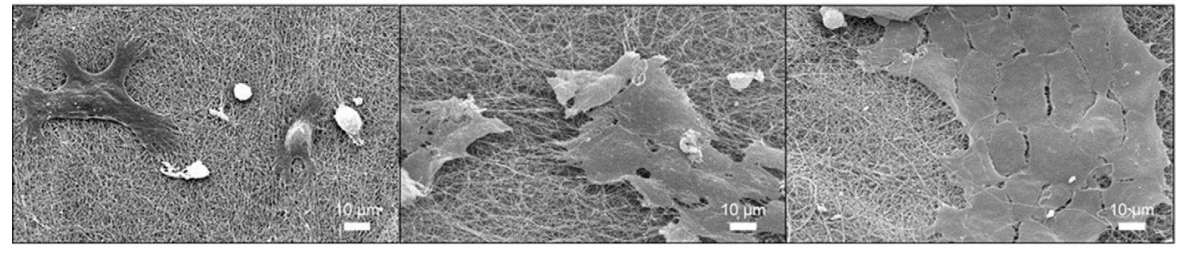

(C)

Fig. 8.7 Scanning electron microscopy observation of human fibroblasts (A), microvascular endothelial cells (B), and keratinocytes (C) cultured on electrospun chitosan nanofibers for 1, 3, or 7 days.

Reproduced with permission from Tchemtchoua VT, Atanasova G, Aqil A, Filée P, Garbacki N, Vanhooteghem O, et al. Development of a chitosan nanofibrillar scaffold for skin repair and regeneration. Biomacromolecules 2011;12:3194-204 with permission from American Chemical Society. 
excised wound surface, to absorb the exudates, and to be fully biocompatible. The control wound beds exhibited the presence of inflammatory cells in a poorly organized tissue; in contrast, a progressive remodeling of the granulation tissue could already be observed in chitosan-treated wounds. At day 14, a well-developed vascular network was also present in the chitosan-treated wounds, and on day 21 , most of the initial wound bed is replaced by a well-vascularized newly formed skin that has been not observed with control animals.

Silk fibroin (SF) is a typical fibrous protein, mainly produced by silkworm. Recently, significant attention has been turned toward SF in wound healing applications since fibroin has the unique properties of skin regeneration including excellent biocompatibility, enhanced collagen biosynthesis, minimal immunogenicity, antiinflammatory activity, hemostatic activity reepithelialization, and elimination of scarring. Till now, few attempts have been made on the electrospun SF scaffolds in wound healing applications. Sheikh et al. [35] prepared 3-D SF nanofibrous scaffolds using cold-plate electrospinning (CPE) to overcome the limitations of the traditional electrospinning (TE) and salt leaching electrospinning (SLE). The prepared 3-D SF nanofibrous scaffolds were used to develop the skin substitute by coculturing two different cell lines in air-liquid culture system. Fig. 8.8A shows the schematic illustration of air-liquid culture system, consisting of keratinocytes cocultured with fibroblasts in the presence of 3-D nanofiber scaffolds produced by $50 \%$ of humidity during CPE. The results of incubation period 6,7 , and 8 weeks in the presence of 3-D nanofibers are presented in Fig. 8.8B-D, which demonstrated as the time of incubation passes; a number of fibroblasts and keratinocytes that are infiltrating into 3-D nanofiber scaffolds were increased. And also, the fibroblasts were observed in deep layers whereas keratinocytes at superficial layer, and it resembles the artificial dermis. And also, collagen-like ECM is predominately present in deeper layer of 3-D nanofiber scaffolds at 8 weeks by Masson's trichrome (MT) stain (Fig. 8.8E).

A recent study by $\mathrm{Ju}$ et al. [36] reported fabrication of SF nanomatrix by electrospinning and evaluated as wound dressing material in a burn rat model. In brief, the SF solution was mixed with polyethylene oxide (PEO) to obtain desirable viscosity and spinnability. A detailed description of the preparation procedure is illustrated in Fig. 8.9. The prepared SF electrospun nanomatrix was applied on the burned wound created on the back of SD rats to study the effect of SF nanomatrix in wound healing process as compared with medical gauze and MEDIFOAM (polyurethane hydrocelluar dressing foam). The wound size reduction, histological examination, and the quantification of transforming growth factor (TGF)- $\beta 1$ and interleukin (IL)- $1 \alpha, 6$, and 10 were considered as parameters in evaluating healing effects. Fig. 8.10 indicated that at day 28 , the areas of wounds treated with SF nanomatrix and MEDIFOAM decreased to $4 \%$ and $8 \%$, respectively, whereas the wound size of the medical gauze-treated group remains $18 \%$.

Further, the histological analysis indicated that the deposition of collagen in the dermis was organized by covering the wound area in the SF nanomatrix-treated group. And also, the expression level of proinflammatory cytokine (IL-1 $\alpha$ ) was significantly reduced on day 7 and increased expression of TGF- $\beta 1$ in the wound treated with SF nanomatrix noted on 21 days posttreatment as compared with gauze-treated group 


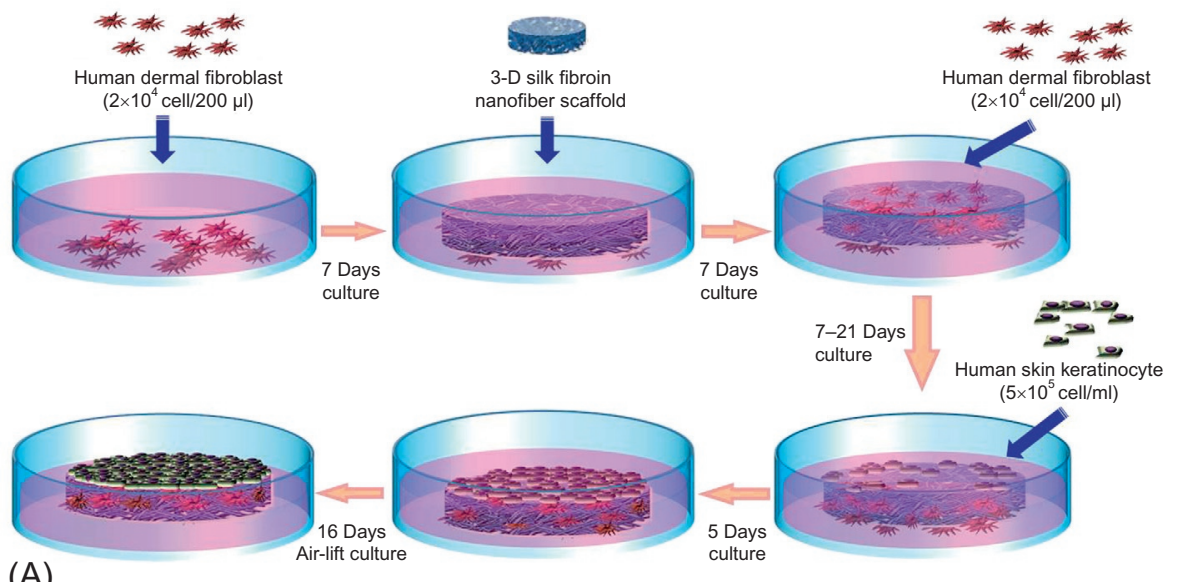

(A)

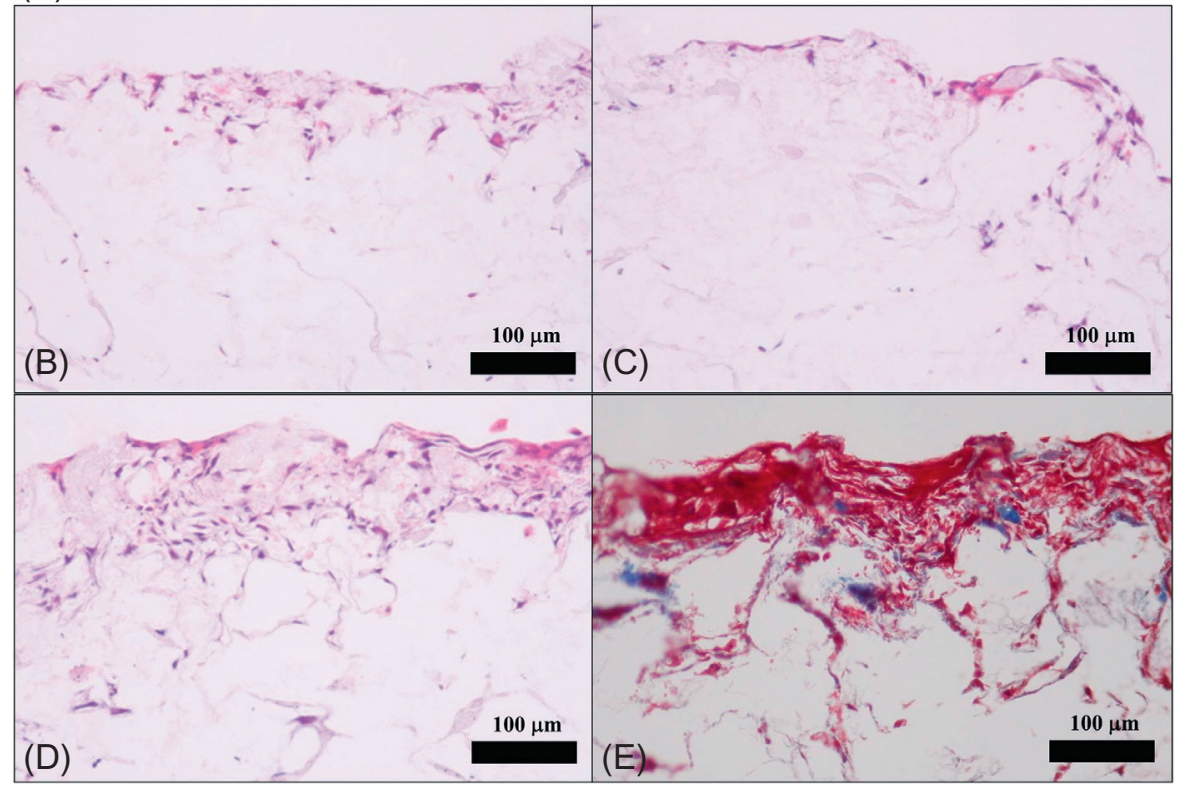

Fig. 8.8 Schematic illustration for cocultured method (fibroblasts and keratinocytes) using air-liquid culture system (A). Histological appearance after coculturing of 3-D nanofibers scaffolds prepared via $50 \%$ humidity by $\mathrm{CPE}$ technique. The H\&E staining results after coculturing for 6 weeks (B), H\&E staining results after coculturing 7 weeks (C), and H\&E staining results after coculturing 8 weeks (D). The results of MT staining after coculturing of fibroblasts and keratinocytes for 8 weeks (E).

Reproduced with permission from Sheikh FA, Ju HW, Lee JM, Moon BM, Park HJ, Lee OJ, et al. 3D electrospun silk fibroin nanofibers for fabrication of artificial skin. Nanomedicine 2015;11:681-91 with permission from Elsevier. 


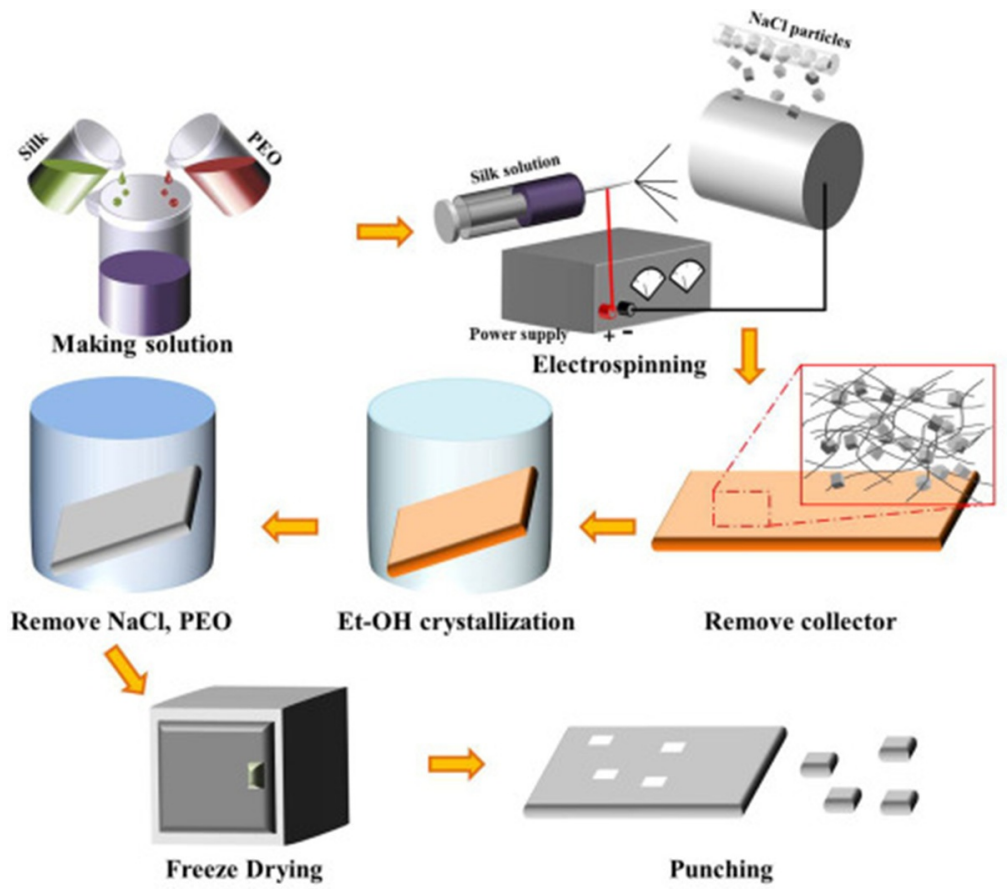

Fig. 8.9 Fabrication of the SF electrospun nanomatrix. A schematic depiction of the $\mathrm{NaCl}$ addition during electrospinning and crystallization.

Reproduced with permission from Ju HW, Lee OJ, Lee JM, Moon BM, Park HJ, Park YR, et al. Wound healing effect of electrospun silk fibroin nanomatrix in burn-model. Int J Biol Macromol 2016;85:29-39 with permission from Elsevier.

that revealed the enhanced wound healing property of SF nanomatrix. Likewise, many other nanofibers produced from natural polymers were used in wound healing applications.

Incorporation of active agents into polymeric nanofibers is always of great interest in improved biomedical applications. To date, a variety of bioactive agents including antimicrobial agents, growth factors, genes, vitamins, metal, and metal oxide nanoparticles have been introduced into polymeric nanofibers for enhanced wound healing [12,21,22,26]. A recent study by Li et al. [37] demonstrated the incorporation of vitamin A palmitate (VA) and vitamin E TPGS (VE), common derivatives of the unstable vitamins $\mathrm{A}$ and $\mathrm{E}$ into biodegradable GE nanofibers using electrospinning. The prepared nanofibers were studied for their sustained release behavior, antibacterial activity, cell proliferation, and in vivo wound healing performances. Both VA and VE were mixed with GE solution further electrospun to obtain nanofibers. The in vitro study results indicated that both vitamins have similar release profile, addition of VE showed improved antibacterial efficiency, and VA supports 
Fig. 8.10 (A) Burn wound area on rat skin right after the creation. (B) Residual wound area change with healing time (28 days). (C) Gross findings of wound area treated with different wound dressing materials $(\mathrm{C}$, medical gauze; $\mathrm{S}, \mathrm{SF}$ nanomatrix; and $\mathrm{M}$, MEDIFOAM).

Reproduced with permission from Ju HW, Lee OJ, Lee JM, Moon BM, Park HJ, Park YR, et al. Wound healing effect of electrospun silk fibroin nanomatrix in burn-model. Int J Biol Macromol 2016;85:29-39 with permission from Elsevier.
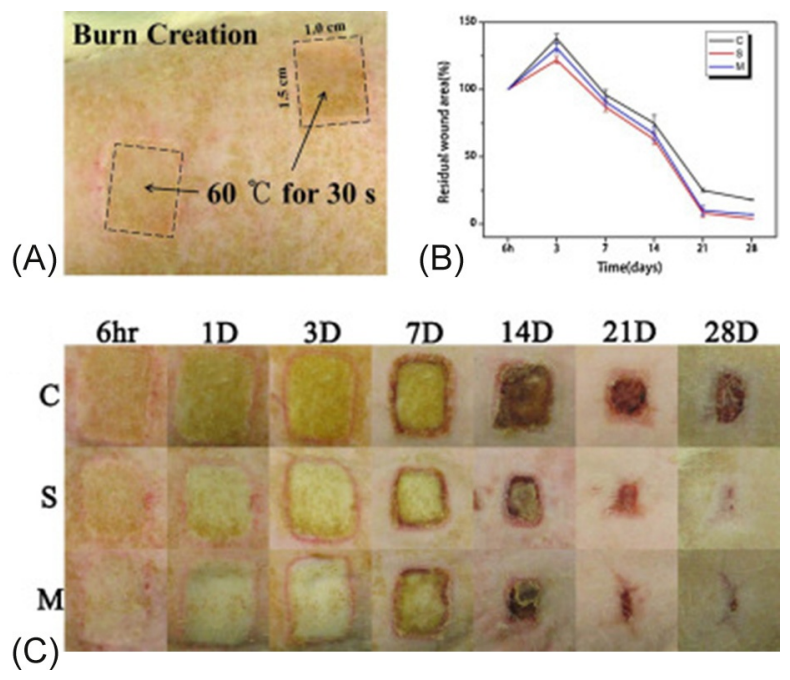

better fibroblast cell proliferation. The in vivo wound healing study was performed in SD rats, and the efficiency of wound healing property was compared with commercial antiseptic gauze loaded with ampicillin, gauze soaked with a vitamin solution, GE fibers, and GE films loaded with VA and VE. Fig. 8.11 depicts representative images of wounds made to the skin of male rats at various times after infliction. Throughout the healing time, the areas of wounds treated by the commercial gauze or the gauze immersed in vitamin solution were larger than those treated with the fibers or the film. Compared with the GE fibers and gelatin/VA/VE $(\mathrm{G} / \mathrm{A}+\mathrm{E})$ film, the wound areas of the $\mathrm{G} / \mathrm{A}+\mathrm{E}$ fiber-treated rats were smaller, indicating a better healing performance. The histological studies were also in agreement with the above findings, which illustrate the incorporation of vitamin in GE nanofibers enhanced the wound healing performances.

Silver nanoparticles (AgNPs) have been well recognized for their excellent antimicrobial efficiencies against wide range of pathogens. A considerable effort has been made to incorporate AgNPs in electrospun nanofibers for wound healing applications. Rath et al. [38] prepared AgNPs-incorporated collagen nanofibers and further investigated their antibacterial activity against potential pathogens and in vivo wound healing performance using Wistar rats. The antibacterial study suggested that AgNP composite nanofibers showed excellent activity against Staphylococcus aureus and Pseudomonas aeruginosa. In contrast, no inhibition potential against pathogens was noted with plain collagen nanofibers. The antibacterial study result clearly reveals that the AgNP composite nanofibers are capable of providing an aseptic environment at the wound site. The wound healing study also resulted 
(A)
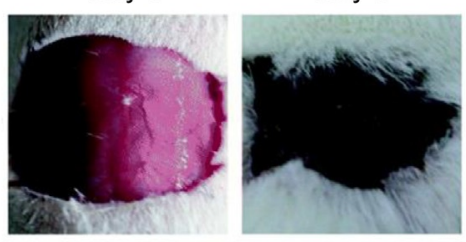

Day 14
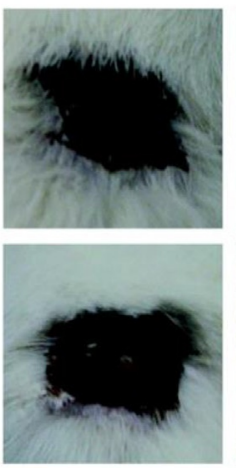

(B)

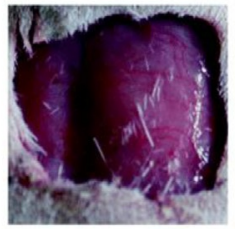

(C)
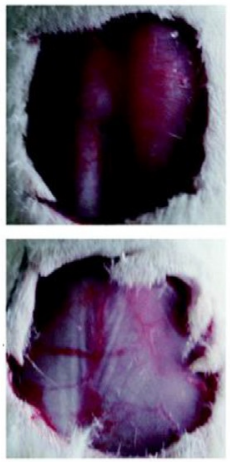

(D)
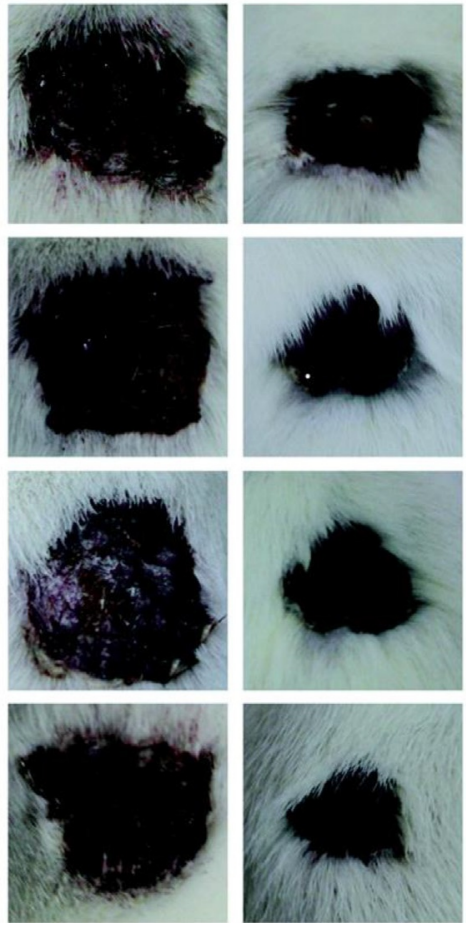
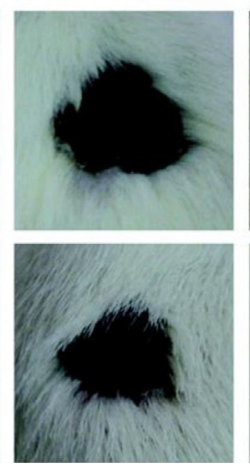

Day 21
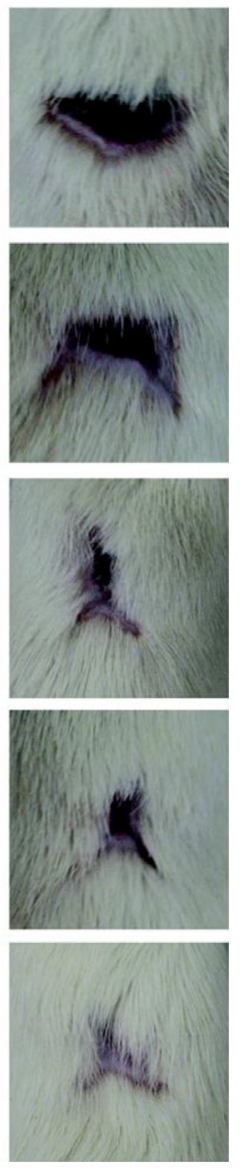

Fig. 8.11 Representative images of the skin wound recovery process after $0,5,14$, and 21 days. The wound surfaces were treated with (A) commercial gauze, (B) the same type of gauze soaked with a vitamin solution (1.4\% w/v VE and $0.14 \% \mathrm{w} / \mathrm{v} \mathrm{VA}),(\mathrm{C})$ cross-linked gelatin (G) fibers (D) cross-linked gelatin films loaded with VA and VE, and (E) cross-linked gelatin fibers loaded with VA and VE $(\mathrm{G} / \mathrm{A}+\mathrm{E})$.

Reproduced with permission from Li H, Wang M, Williams GR, Wu J, Sun X, Lv Y. Electrospun gelatin nanofibers loaded with vitamins $\mathrm{A}$ and $\mathrm{E}$ as antibacterial wound dressing materials. RSC Adv 2016;6:50267-77 with permission from Royal Society of Chemistry.

in faster wound contraction than plain collagen nanofibers. Similarly, titanium dioxide $\left(\mathrm{TiO}_{2}\right)$ nanoparticles were incorporated into SF nanofibers. The nanofibers were studied for their hemocompatibility, cytocompatibility, cell spreading, and antibacterial activity. The altogether results showed that the prepared nanofibrous membrane $\mathrm{TiO}_{2}$ nanoparticles possess good biocompatibility, cell adhesion, and antimicrobial potential, which further suggest the possible utilization for wound dressing [39]. 
In recent years, a significant attention has been paid toward antimicrobial peptides (AMPs) as alternative antimicrobial materials. In another study, antimicrobial peptide motif (Cys-KR12) originating from human cathelicidin peptide (LL37) was immobilized onto electrospun SF nanofiber membranes for wound care purposes. The study reported that Cys-KR12-immobilized SF nanofiber membrane exhibited antibacterial potential against S. aureus, S. epidermidis, Escherichia coli, and $P$. aeruginosa. The fibrous membrane also facilitated proliferation of keratinocytes and fibroblasts. Further, it suppressed the lipopolysaccharides (LPS)-induced TNF-a expression of monocytes, suggesting as a promising candidate in wound dressing application [40].

\subsubsection{Synthetic polymer nanofibers as wound healing scaffolds}

Synthetic polymers provide many advantages over natural polymers, most importantly ability to tailor for better mechanical and degradation properties by altering chemical composition, molecular weight, copolymerization, crystallinity, etc. Aliphatic polyesters are common biodegradable synthetic polymers that offer biomedical applications because of their mechanical strength, adjustable properties, their nontoxic degradation products and processability. There are many synthetic polymers including polyglycolic acid (PGA), poly(lactic acid) (PLA), and polycaprolactone (PCL) that have been approved by US Food and Drug Administration (FDA) for their applications in tissue engineering [41]. The polymers including PLA, PCL, poly(lactic-co-glycolic acid) (PLGA), PEO, polyurethane (PU), and poly(vinyl alcohol) (PVA) have been widely used in wound healing applications [17,21,26].

Synthetic polymeric nanofibers are generally used in wound healing applications by functionalizing with bioactive agents [42]. PCL is a hydrophobic polyester and widely used in tissue engineering applications upon incorporating various bioactive agents. Bahrami et al. [43] prepared unrestricted somatic stem cells (USSCs) loaded in PCL nanofibrous scaffolds and used for skin regeneration on full-thickness skin defects of rats. The wound healing study in animal model exhibited noticeable effect on wound closure on 21 days of treatment. The reconstructed skin area treated with nanofibrous scaffold loaded with USSCs has shown an intact epithelium together with the formation of hair follicles and sebaceous glands. Similarly, zinc oxide $(\mathrm{ZnO})$ nanoparticles have been incorporated into PCL nanofibers using electrospun nanofibers, and further, their ability to perform as skin substitute materials was tested using American satin guinea pigs [44]. The in vivo fibroblast cell proliferation was analyzed during 20 days of subcutaneous implantation of neat PCL membranes and PCL membranes containing $1 \mathrm{wt} \% \mathrm{ZnO}$ nanoparticles. The histological image indicated that the $\mathrm{ZnO}$ nanoparticle-impregnated PCL nanofibrous scaffolds enhanced the cell proliferation as shown in Fig. 8.12. Further, the wound healing study is also following a 30-day observation period; the wounds were completely closed without any sign of scar formation for nanoparticle incorporated membranes. Conversely, hair formation on healed area was significantly less for the animals treated with neat PCL membranes. The overall results indicated that the incorporation of $\mathrm{ZnO}$ nanoparticles notably enhanced the wound healing effects. And also, in another 


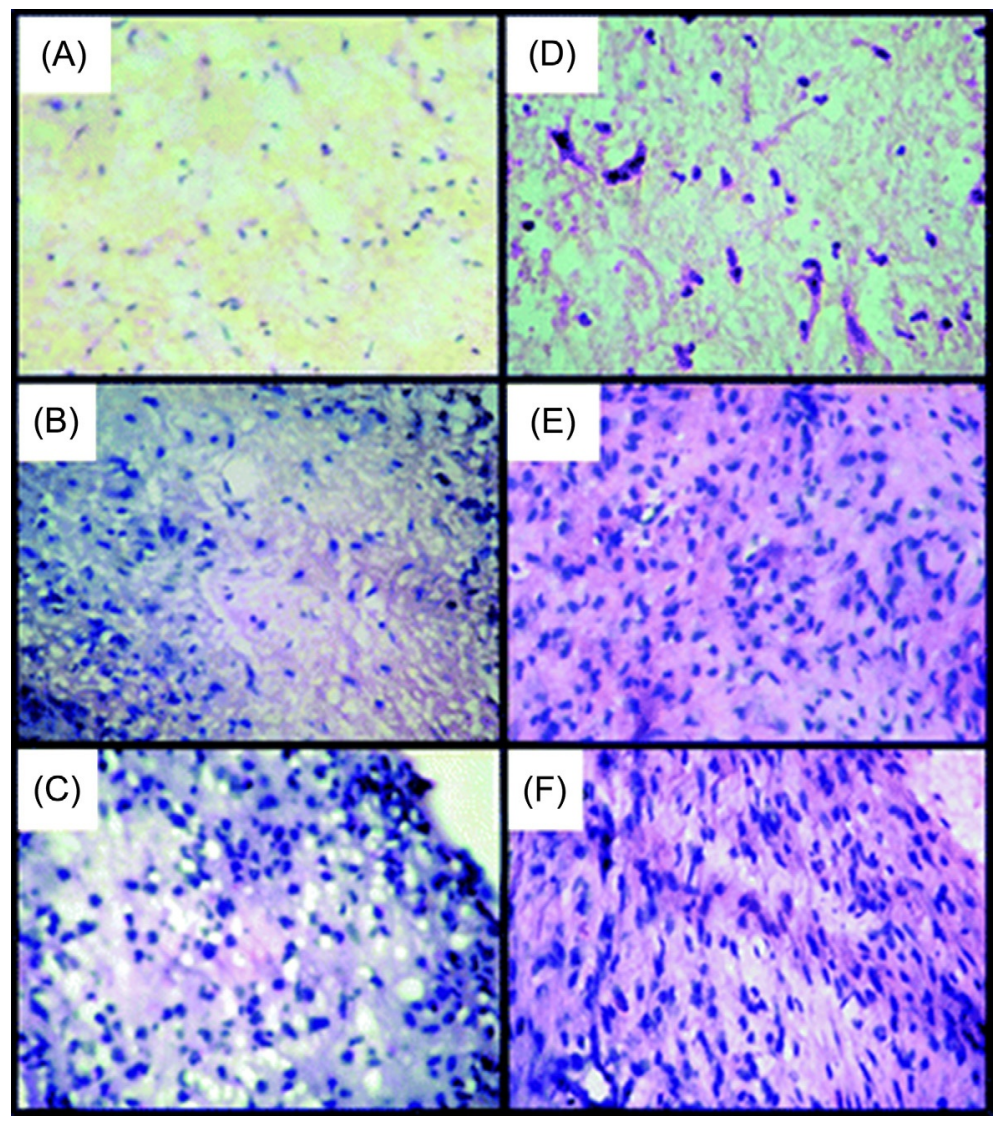

Fig. 8.12 Histological images (H\&E staining) of neat PCL membranes (A-C) and PCL membranes containing $1 \mathrm{wt} \% \mathrm{ZnO}$ nanoparticles (D-F) after 5 days (A and $\mathrm{D}$ ), 10 days (B and $\mathrm{E})$, and 20 days ( $\mathrm{C}$ and $\mathrm{F}$ ) of subcutaneous implantation in guinea pigs.

Reproduced with permission from Augustine R, Dominic EA, Reju I, Kaimal B, Kalarikkal N, Thomas S. Electrospun polycaprolactone membranes incorporated with $\mathrm{ZnO}$ nanoparticles as skin substitutes with enhanced fibroblast proliferation and wound healing. RSC Adv 2014;4:24777-85 with permission from The Royal Society of Chemistry.

recent study reported by Pinzón-García et al. [45], Bixin (Bix), an antioxidant carotenoid pigment, was loaded to PCL nanofibers, and the in vivo wound healing study in diabetic mice resulted in accelerated wound healing and in reduced scar formation as compared with pure PCL nanofibers.

PLA is an aliphatic biodegradable and compostable thermoplastic polymer derived from renewable plant sources and has generated considerable interest in wide range of biomedical applications. Nguyen et al. [46] incorporated curcumin (Cur) into PLA nanofibers for wound healing applications because of their multibiological functions including antiinflammatory, antioxidant, antitumor, and wound healing properties. 
Cell attachment study showed the improved cell proliferation and attachment for Cur-loaded PLA nanofibers. Similarly, the wound healing study in C57BL/6 mice demonstrated enhanced wound healing property for Cur-loaded PLA nanofibers $(99 \%)$ on day 15 , which is significantly higher than the wound treated with gauze and PLA nanofibers as shown in Fig. 8.13.

PLGA is the most extensively used biodegradable and biocompatible polymer in therapeutic delivery and tissue engineering. Further, PLGA forms nontoxic end products, increases wettability, and promotes cell proliferation. Lee et al. [47] used PLGA polymer for incorporating metformin, an antihyperglycemic agent usually prescribed for treating type 2 diabetes. The prepared metformin-incorporated PLGA nanofiber was investigated to treat wounds in diabetic SD rat model. The biodegradable nanofibers were shown to release high concentration of metformin over three weeks' time and had more water-containing capacity than virgin PLGA fibers. The wound healing study outcome clearly demonstrated the enhanced wound healing and reepithelialization for the PLGA fibers incorporated with metformin than the animals

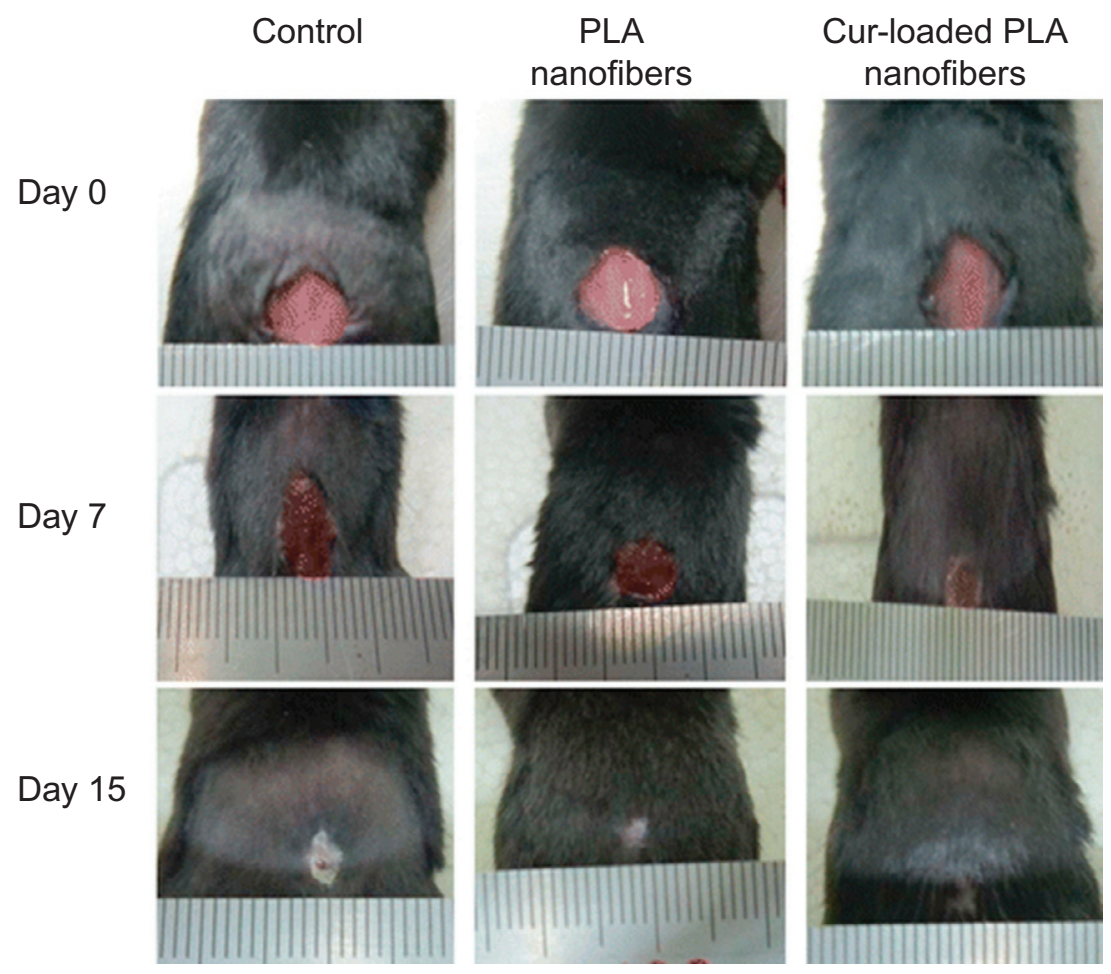

Fig. 8.13 Photographs of the closure of control, PLA nanofiber-treated and Cur-loaded PLA nanofiber-treated mouse back wounds on days 0,7 , and 15 .

Reproduced with permission from Nguyen TTT, Ghosh C, Hwang SG, Tran LD, Park JS. Characteristics of curcumin-loaded poly(lactic acid) nanofibers for wound healing. J Mater Sci 2013;48:7125-33 with permission from Springer. 

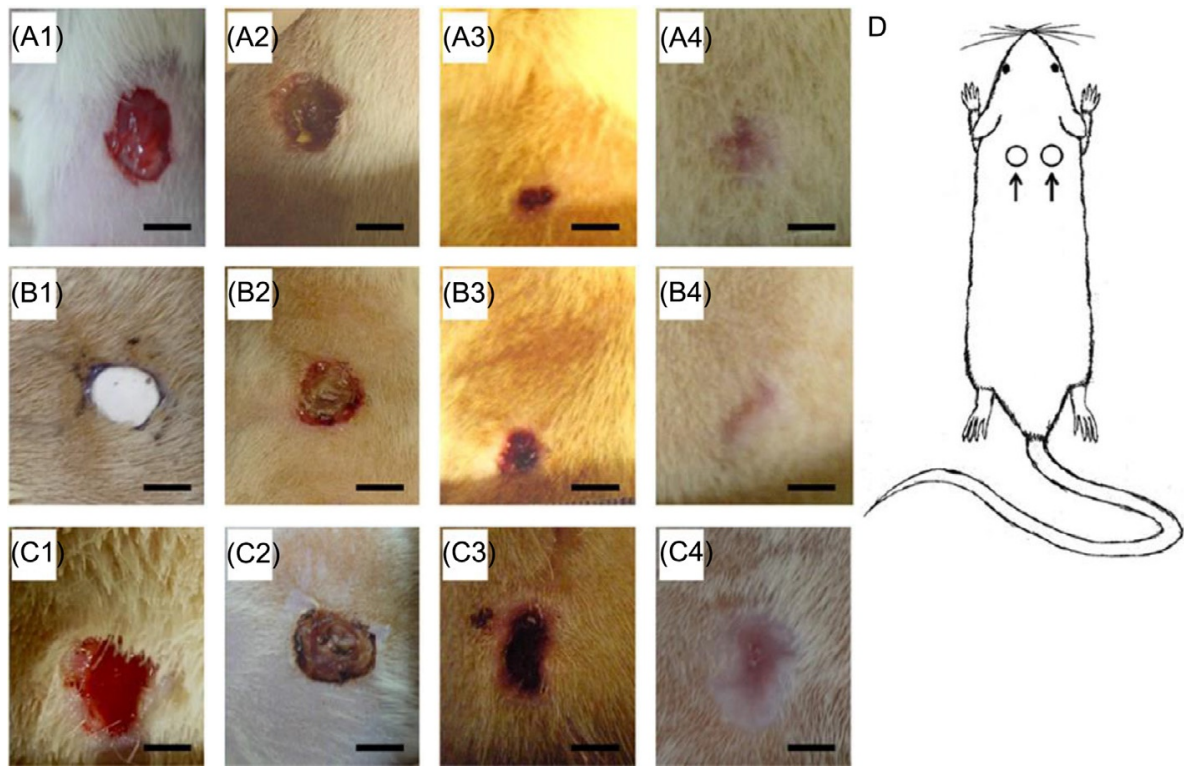

Fig. 8.14 Appearance of healing wound on days 0 (1), 3 (2), 7 (3), and 14 (4) after treatment: (A) PLGA with metformin group and (B) virgin PLGA (C) conventional gauze sponge group (two circular wounds with diameter of $8 \mathrm{~mm}$ were prepared on back of each rat, as shown in (D)) (scale bar $=5 \mathrm{~mm}$ ). Group A exhibited faster healing than group B and group $\mathrm{C}$ (post hoc $P<.05)$.

Reproduced with permission from Lee CH, Hsieh MJ, Chang SH, Lin YH, Liu SJ, Lin TY, et al. Enhancement of diabetic wound repair using biodegradable nanofibrous metformin-eluting membranes: in vitro and in vivo. ACS Appl Mater Interfaces 2014;6:3979-86 with permission from American Chemical Society.

treated with virgin PLGA nanofibrous membrane and conventional gauze as shown in Fig. 8.14. Similarly, fusidic acid (FA) is an antibiotic that belongs to a group of fusidanes, which has been incorporated into PLGA nanofiber to study the wound healing efficiency in lightly contaminated or S. aureus heavily infected wounds of rat. In either case, FA-loaded fibers have shown speed, quality (minimal scarring), reduced infection dissemination, and fatalities in wound healing than untreated sites [48].

PU is a block of copolymers, widely used in wound dressings because of their good barrier and oxygen permeability properties. Khil et al. [49] produced PU electrospun nanofibers and investigated their wound healing performance in guinea pigs. The PU nanofibers found to have good and immediate adherence property to wet wound surface. The wound treated with PU nanofibers had several characteristic findings compared with control animals in which severe infiltration of inflammatory cells and thick scab were observed. The PU nanofibrous membrane was uniformly adhered on the wound surface without any fluid accumulation. At the end of the study, the wounds covered with PU electrospun nanofibers showed increased rate of epithelialization and 
well-organized dermis as compared with TEGADERM, a commercial wound healing material. Another study reported the preparation of PU nanofibers by blending emu oil that is composed of fatty acids and possesses analgesic and antiinflammatory effects. The results indicated that the emu oil contained PU nanofibers that enhanced cell viability and proliferation of 3T3-L1 fibroblasts cells. The membrane as well showed good bacteriostatic activity against both Gram-positive and Gram-negative pathogens and expected to have application in wound skin tissue engineering [50]. Similarly, ampicillin-loaded PU (A-PU) nanofibers were electrospun and have shown to maintain good viability in human keratinocyte (HaCaT) cells and exhibit good antibacterial activity against Gram-positive $S$. aureus and Gram-negative $K$. pneumonia. The overall outcome suggests that A-PU scaffolds can be used in wound healing and infection control applications [51]. Likewise, incorporation of AgNPs in PU nanofibers were also reported to show enhanced performances and suggesting for their use in wound healing applications [52,53].

PVA is a good biocompatible polymer used in variety of advanced biomedical applications. Nguyen et al. [54] reported the preparation of AgNPs-loaded PVA electrospun fibers by coupling microwave and electrospinning methods and further studied their antibacterial activity against E. coli (Gram-negative) and S. aureus (Gram-positive) bacteria for intended use in wound healing applications. The silver nitrate containing PVA solution was microwave irradiated and then electrospun for obtaining AgNPs-loaded PVA electrospun fibers. The antibacterial stud revealed no obvious zones of inhibition observed for pure PVA membrane, whereas AgNPs-loaded PVA electrospun membrane heated $150^{\circ} \mathrm{C}$ showed strongest antimicrobial activities against both Gram-positive S. aureus and Gram-negative E. coli; however, $S$. aureus is more sensitive than the $E$. coli bacteria. The fabricated mat that had a high tensile stress and antimicrobial potential has beneficial for skin applications.

Poly-L-lactic acid (PLLA) is a biodegradable poly( $\alpha$-hydroxy acids) and has been widely investigated for their use in treatment of musculoskeletal injuries, controlledrelease systems, and tissue engineering. A recent study by $\mathrm{Li}$ et al. [55] reported recombinant silkworm AMP Bmattacin2-incorporated PLLA exhibited broad spectrum of antibacterial activity and selective killing ability toward skin and colon cancer cells over their normal cell counterparts. The antibacterial study using AATCC100 against E. coli and $S$. aureus indicated that PLLA/Bmattacin2 had 26.2\% inhibition on $E$. coli while it had $32.3 \%$ inhibitive activity on $S$. aureus as shown in Fig. 8.15A. Further, live/dead assay by SYTO 9 and propidium iodide indicated the live (green) and dead bacteria (red) on fabricated membranes (Fig. 8.15b1 and b3). Further, SEM observations of both bacteria showed the morphology of bacteria has been destroyed with PLLA/Bmattacin2 treatment as shown in Fig. 8.15b6 and c6, respectively. Further, cell proliferation assay with human skin cell HFF1 demonstrated the increased cell proliferation rate on both PLLA and PLLA/Bmattacin2 at day 1 , day 3 , and day 7 showing their cell compatibility nature. The SEM and fluorescent staining also confirmed the cell spreading over the surface of the membranes. Therefore, the prepared PLLA/Bmattacin 2 can be used in skin reconstruction, wound dressing, and healing. 


\begin{tabular}{cccc}
\hline & E.coli & \multicolumn{2}{c}{ S.aureus } \\
\hline PLLA & PLLA/Bmattacin2 & PLLA & PLLA/Bmattacin2 \\
& & & \\
$1.84 \pm 0.09 \times 10^{2}$ CFU & $1.34 \pm 0.19 \times 10^{2}$ CFU & $2.0 \pm 0.24 \times 10^{2}$ CFU & $1.35 \pm 0.08 \times 10^{2}$ CFU \\
$\begin{array}{l}\text { Antimicrobial } \\
\text { activity } \%)\end{array}$ & 26.2 & & 32.3 \\
\hline
\end{tabular}

\section{(A)}
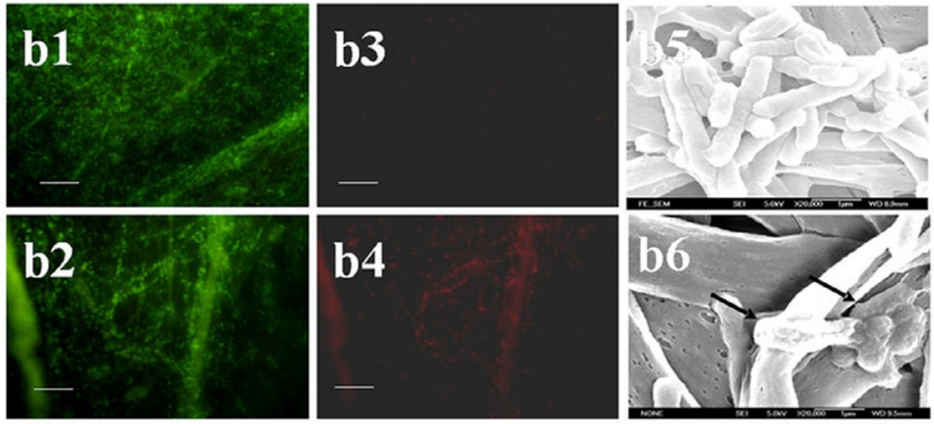

PLLA

(B)
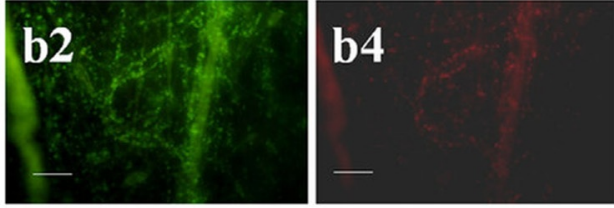

PLLA/

Bmattacin2
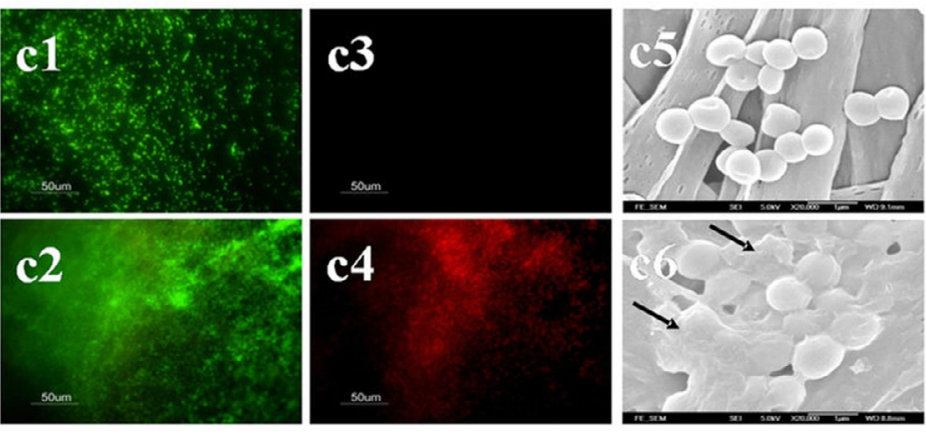

PLLA

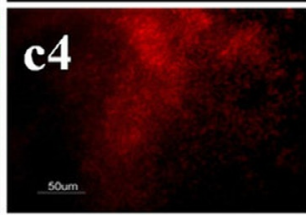

\section{PLLA/}

Bmattacin2

(C)

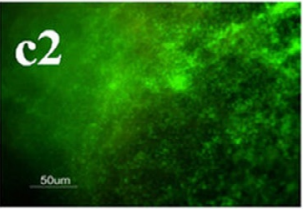

Fig. 8.15 Antibacterial effect of electrospun composite membranes. (A) Investigating antibacterial effect of electrospun composite membranes on $E$. coli and $S$. aureus by AATCC100 method. (B) Observation of E. coli cultured on the surface of PLLA or PLLA/ Bmattacin2 membranes. (b1-b4) Live/dead bacterial staining. (b5 and b6) SEM observation. (C) Observation of $S$. aureus cultured on the surface of PLLA or PLLA/Bmattacin 2 membranes. (c1-c4) Live/dead bacterial staining. (c5 and c6) SEM observation.

Reproduced with permission from Li Z, Liu X, Li Y, Lan X, Leung PH, Li J, et al. Composite membranes of recombinant silkworm antimicrobial peptide and poly(L-lactic acid) (PLLA) for biomedical application. Sci Rep 2016;6:31149 with permission from Nature Publishing Group.

\subsubsection{Composite polymer nanofibers as wound healing scaffolds}

Composite polymeric nanofibers are produced by the combination of natural and/or synthetic polymers that possess advantages of each polymer to overcome the limitations and aiming wound healing applications. There have been a number of studies reported for the preparation of different polymeric nanofibers and incorporation of 
active agents for improved wound healing performances. Ebrahimi-Hosseinzadeh et al. [56] reported preparation of nanofibrous scaffold by blending two natural polymers, namely, GE and HA. In vivo wound healing study in Wistar rats with deep second-degree burns has been investigated in comparison with ChitoHeal gel (a commercial wound dressing). Following 14 days of treatments with GE/HA, composite nanofibrous membranes and ChitoHeal gel reached up to wound closure of $81.9 \%$ and $77.8 \%$, respectively, while untreated control has shown $65 \%$ wound closure efficiency. The histopathologic study conducted on postoperative days of 7 and 14 exhibited more epidermis formation in the gel and scaffold groups. And also, a number of inflammatory cells observed in these groups were significantly lesser than the control group.

Application of herbal extracts is familiar in treating burns and wounds since ancient times. Recently, extracts of Cleome droserifolia (CE) and Allium sativum (AE) have been incorporated into honey, poly(vinyl alcohol), chitosan nanofibers (HPCS) to develop biocompatible antimicrobial nanofibrous wound dressing [57]. The extracts were incorporated into HPCS separately, HPCS-CE and HPCS-AE, or in combination, HPCS-CE/AE. The in vitro antibacterial study against $S$. aureus, E. coli, methicillin-resistant $S$. aureus (MRSA), and multidrug-resistant $P$. aeruginosa was carried out in comparison with AquacelAg, a commercial wound dressing, and the study revealed HPCS-AE and HPCS-AE/CE nanofiber mats have shown complete inhibition of $S$. aureus, and the HPCS-AE/CE exhibited mild antibacterial activity against MRSA. Further, the developed nanofiber dressings and the AquacelAg commercial dressing were used in wound healing study on experimental mice having an excisional $9 \mathrm{~mm}$ wound on the dorsal back.

Photographs of the wound region were recorded on days $3,5,7,10$, and 12 to determine the change in the wound size over time (Fig. 8.16). It was observed that the wound closure was greatly enhanced with the HPCS nanofiber mats, and upon addition of AE in the HPCS-AE mats, the wound closure rate increased. On the other hand, the wound closure rate was reduced upon addition of the CE to the HPCS-CE nanofibrous mats, whereas the combination of both extracts within the HPCS-AE/ CE nanofiber dressings showed wound closure rates similar to those of the HPCS dressing. Upon comparing the wound closure rate of the developed nanofibrous dressings to the commercial AquacelAg, it was observed that the HPCS and the HPCS-AE/ CE showed similar effects, whereas the HPCS-AE showed enhanced wound closure rates. Therefore, the antibacterial and wound healing properties of the developed nanofiber mats and their minimal side effects make them competitive candidates for use as effective wound dressings.

Hajiali et al. [58] incorporated lavender oil (LO) into sodium alginate and PEO (SA-PEO/LO) nanofibers and demonstrated their efficiency in antibacterial and treatment of skin burns induced by midrange ultraviolet radiation (UVB). The LO release measurement indicated that therefore, the produced alginate-based nanofibers were active for more than 2 days and able to release the antibacterial and antiinflammatory agent. The antibacterial study against $S$. aureus revealed SA-PEO/LO nanofibers inhibited the proliferation of the microorganisms and inhibition zones with an average diameter of $(21.7 \pm 1.6) \mathrm{mm}$. A desired portion of C57BL/6J mice skin was exposed to 


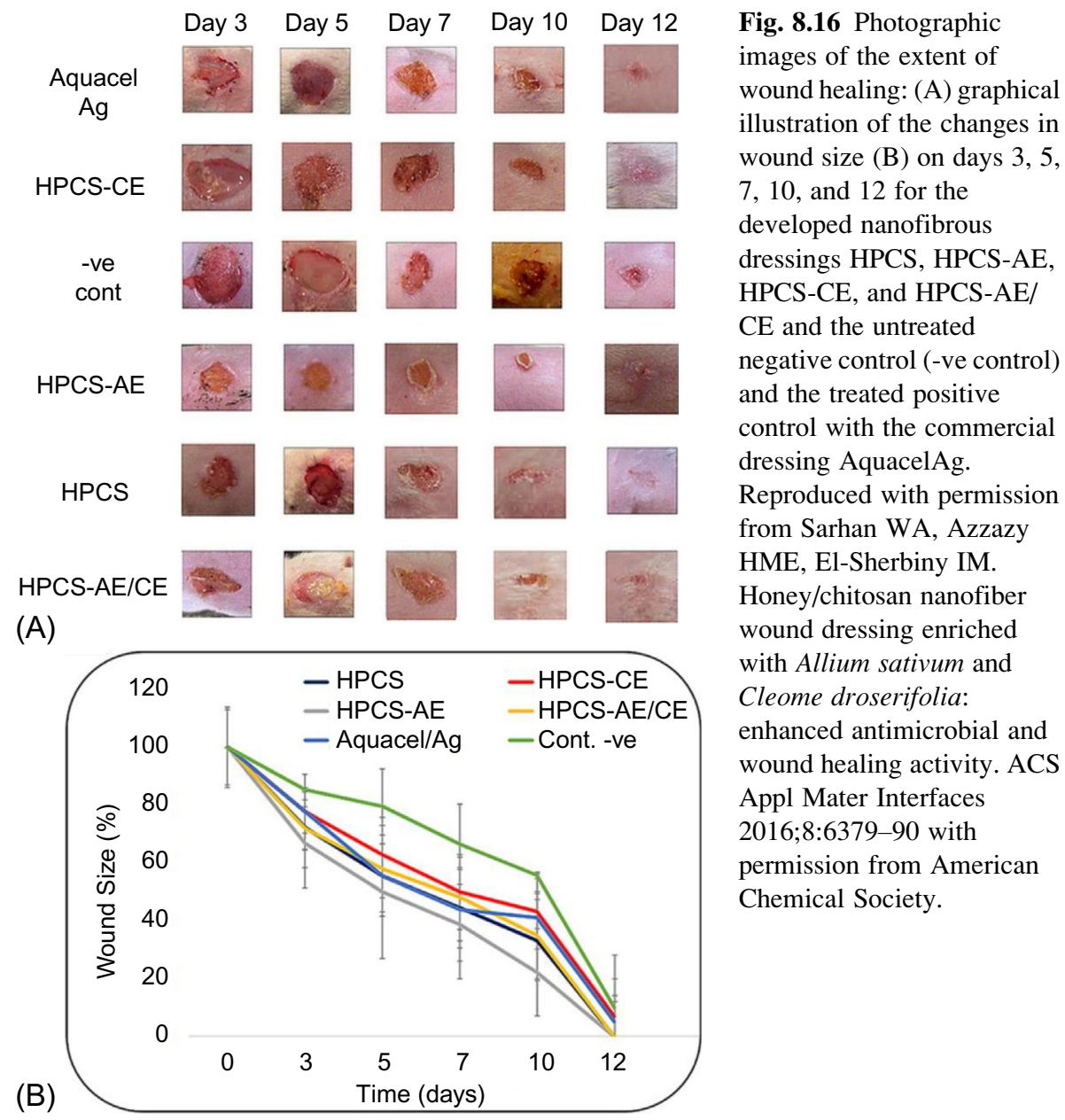

narrowband UVB light source and immediately covered with SA-PEO or SA-PEO/LO nanofibers or a commercial alginate product used as standard of care (3M Tegaderm Alginate). The wound healing and cytokine expression studies have shown that alginate-based nanofibers controlled UVB-induced inflammation; therefore, the electrospun dressings were proved appropriate for the management of burn wounds.

In another study, curcumin-loaded poly( $\varepsilon$-caprolactone) (PCL)/gum tragacanth (GT) (PCL/GT/Cur) nanofibers were investigate for their wound healing potential in diabetic rats. The antibacterial study of the resultant membrane against MRSA and extended spectrum $\beta$-lactamase (ESBL) has shown PCL/GT/Cur nanofibers were $99.9 \%$ antibacterial against MRSA and $85.14 \%$ against ESBL as depicted in Fig. 8.17. Similarly, wound healing study resulted in curcumin-eluting 


\begin{tabular}{|c|c|c|c|c|}
\hline \multirow[b]{2}{*}{ Bacteria } & \multicolumn{2}{|c|}{ Control } & \multicolumn{2}{|c|}{ GT/PVA/cur samples } \\
\hline & MRSA & ESBL & MRSA & ESBL \\
\hline First dilution & & & & \\
\hline Amount & Uncountable & Uncountable & No growth & $6.1 \times 10^{5}(\mathrm{CFU} / \mathrm{ml})$ \\
\hline Second dilution & & & & \\
\hline Amount & Uncountable & Uncountable & No growth & $7.5 \times 10^{5}(\mathrm{CFU} / \mathrm{ml})$ \\
\hline Amount & $3.2 \times 10^{7}(\mathrm{CFU} / \mathrm{ml})$ & $2.8 \times 10^{7}(\mathrm{CFU} / \mathrm{ml})$ & No growth & No growth \\
\hline Average & & & & $6.8 \times 10^{5}(\mathrm{CFU} / \mathrm{ml})$ \\
\hline Antibacterial (\%) & & & $99.9 \%$ & 85.14 \\
\hline
\end{tabular}

Fig. 8.17 Antibacterial activity against MRSA and ESBL for samples containing Cur (average $\mathrm{CFU} / \mathrm{ml})$.

Reproduced with permission from Mohammadi MR, Rabbani S, Bahrami SH, Joghataei MT, Moayer F. Antibacterial performance and in vivo diabetic wound healing of curcumin loaded gum tragacanth/poly( $\varepsilon$-caprolactone) electrospun nanofibers. Mater Sci Eng C 2016;69:1183-91 with permission from Elsevier.

nanofibers improved the wound healing compared with control samples significantly, and they showed complete healing at the surface with well-formed granulation tissue dominated by fibroblast proliferation, collagen deposition, complete early regenerated epithelial layer, and formation of sweat glands and hair follicles. No such appendage formation was observed in the untreated controls during this duration. Further, MT staining confirmed the increased presence of collagen in the dermis of the nanofiber-treated wounds on days 5 and 15, while the control wounds were largely devoid of collagen on day 5 and exhibited less collagen amount on day 15 [59].

Recently, Ganesh et al. [60] demonstrated enhanced wound healing effects of AgNPs-decorated chitosan (CS)-polyvinyl alcohol (PVA) composite electrospun nanofibers, loaded with sulfanilamide. The prepared electrospun nanofibers were systematically studied for in vitro release, antimicrobial, and in vivo wound healing activity. The release profile of drug and AgNPs was fast during initial hours and became relatively slow at later time period as the mean percentage of $99 \%$ in the formulations after $24 \mathrm{~h}$. Further, the composite fibers incorporated with both sulfanilamide and AgNPs revealed enhanced antibacterial performance than the composite fibers incorporated with only sulfanilamide or AgNPs. The in vivo wound healing study in Wistar 
rats demonstrated the enhanced wound healing effects at 14 days for the composite fibers incorporated with sulfanilamide and AgNPs, while control composite nanofiber without active agents and conventional sulfonamide formulation took more than 20 days to recover the wound due to enhanced cell attachment owing to high wettability and positive charge of the composite polymeric system.

Coaxial electrospinning technique has been adopted to prepare asiaticoside mixed chitosan as a core while alginate and PVA as shell nanofibers. Asiaticoside is a major triterpenoid component derived from Centella asiatica (L.) and widely used in antioxidant, antiinflammatory, immunomodulatory, and wound healing applications. In vitro drug release profile showed that alginate/PVA/chitosan coaxial nanofibers are faster and have more drug release rates. A complete burn wound healing performance noted for the alginate/PVA/chitosan coaxial nanofibers is incorporated with $5 \%$ asiaticoside $(\sim 99.2 \%)$ similar to positive control animal treated with asiaticoside cream. The histopathologic investigation also revealed that drug-loaded nanofibers demonstrated extended reepithelialization of desquamated epithelial regions, less inflammatory cell infiltrations, and numerous collagen proliferations and neovasculations as compared with control group. Also, positive expression of vascular endothelial growth factor (VEGF), cluster of differentiation 31 (CD31), proliferating cell nuclear antigen (PCNA), and downregulation of tumor necrosis factor (TNF) and interleukin-6 (IL-6) also validated the improved effect of wound healing [61].

A recombinant platelet-derived growth factor, recombinant human PDGF-BB (rhPDGF-BB)-eluting PLGA-collagen hybrid scaffolds, has been reported to treat diabetic wounds in SD rats [62]. The in vivo and in vitro release of growth factors from the scaffolds was measured using an enzyme-linked immunosorbent assay kit and an elution method. The release profile demonstrated that the nanofibrous scaffold released growth factor for a time period of 21 days. The wound areas treated with rhPDGF-BB-eluting PLGA-collagen hybrid scaffolds (group A), PLGA-collagen hybrid scaffold (group B), and virgin PLGA scaffolds (group C) resulted in the proportions of the wounds areas that were treated by group B and $\mathrm{C}$ fell slowly to $8.3 \% \pm$ $1.6 \%$ and $9.2 \% \pm 0.9 \%$, respectively, by day 14 , whereas rhPDGF-BB-eluting membranes decreased to about $3.6 \% \pm 0.5 \%$ demonstrating promoted wound healing effect as shown in Fig. 8.18. Histopathologic study indicated that after 14 days, rhPDGF-BB-eluting PLGA-collagen hybrid scaffolds exhibited full reepithelialization and the highest proliferation of keratinocytes in the epidermis layer. Also the expression of collagen contents in PLGA-collagen hybrid scaffolds with rhPDGF-BB-eluting group or without rhPDGF-BB-eluting group was considerably higher than that in PLGA-only group with noted indication of collagen content in rhPDGF-BB-eluting PLGA-collagen hybrid scaffolds group, which was remarkably exceeded than that in PLGA-collagen hybrid scaffolds group on day 7 as can be seen from Fig. 8.19.

The electrospun nanofiber-based hydrocolloids and hydrogels are of significant interest in recent years for wound healing applications. Kim et al. [63] recently prepared nanofiber-based hydrocolloid scaffold using thermoplastic polyurethane (TPU)/ sodium carboxymethyl cellulose (SCMC). A wound healing study on the prepared 
(A)
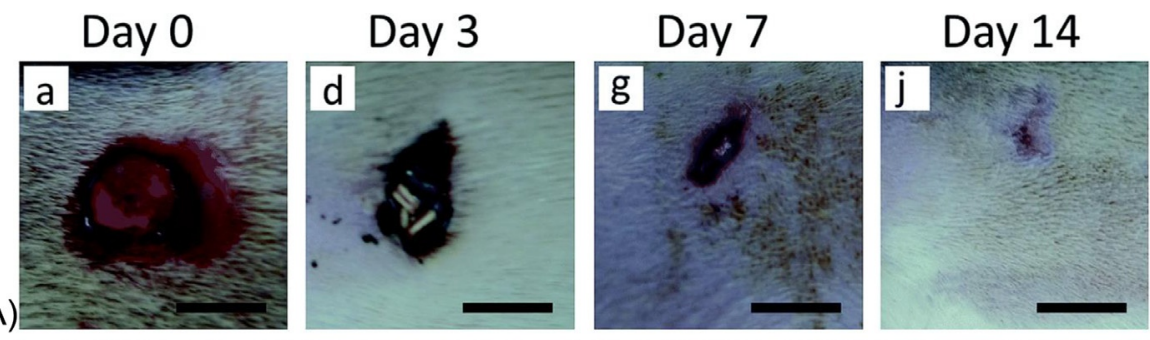

(B)
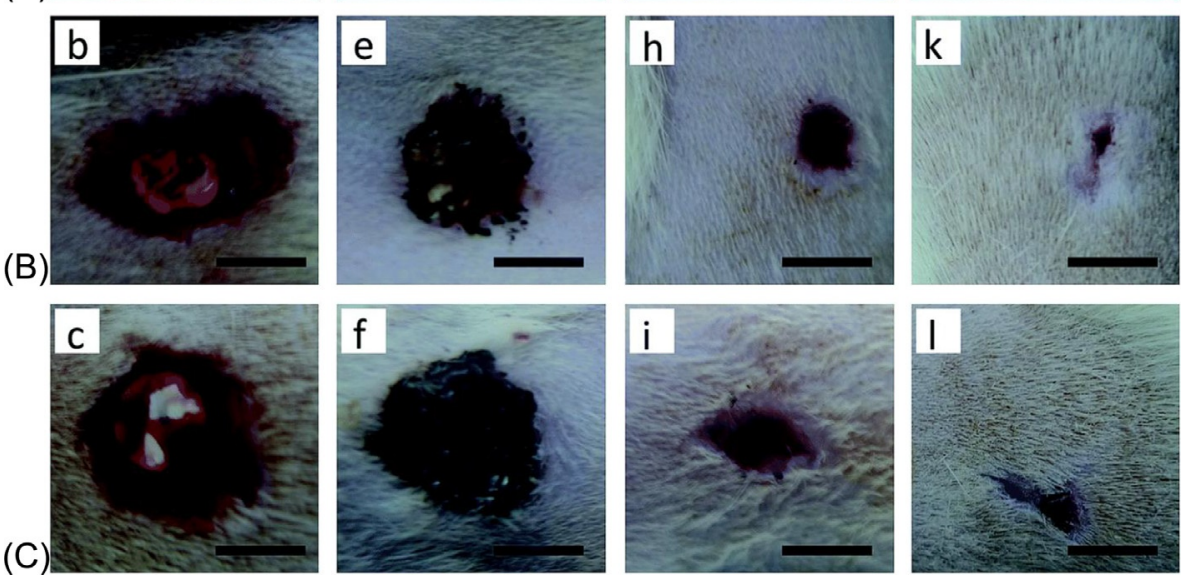

Fig. 8.18 The process of wound repair on different days. Days $0(a-c), 3(d-f), 7(g-i)$, and $14(\mathrm{j}-1)$ following treatment with groups $(\mathrm{A}-\mathrm{C})($ scale bar $=5 \mathrm{~mm})$.

Reproduced with permission from Lee CH, Hsieh MJ, Chang SH, Lin YH, Liu SJ, Lin TY, et al. Enhancement of diabetic wound repair using biodegradable nanofibrous metformin-eluting membranes: in vitro and in vivo. ACS Appl Mater Interfaces 2016;6:3979-86 with permission from The Royal Society of Chemistry.

hydrocolloids has been demonstrated using Institute of Cancer Research (ICR) mice with full-thickness wound for a time period of 21 days. The wound size was observed on days $3,7,14$, and 21 to evaluate the wound healing process. Wound healing in the hydrocolloid fiber-treated group was similar to that of the dried gauze and commercial wound dressing group. However, severe inflammatory reactions were noted in the dried gauze-treated group with reddening and swollen of wounds till 14 days. Conversely, the wound treated with hydrocolloid fibers was found to be neat and moist, without inflammatory exudates, and newly formed epidermis has been noted as similar to commercial wound dressing treatment. The histological evaluation demonstrated enhanced reformation of granulation tissue and reepithelialization over treatment time in all groups. But, a well-organized newly formed epithelium was observed in both commercial dressing and hydrocolloid fiber-treated groups, whereas this is not the case with gauze treatment. Similarly, Xu et al. [64] reported the preparation of chitosan/PLA/PEG hydrogel nanofibers for wound dressing application. The hydrogel nanofibers found to have quick absorption behavior, high equilibrate water absorption, and good air permeability, which may result in absorbing excess exudates, 


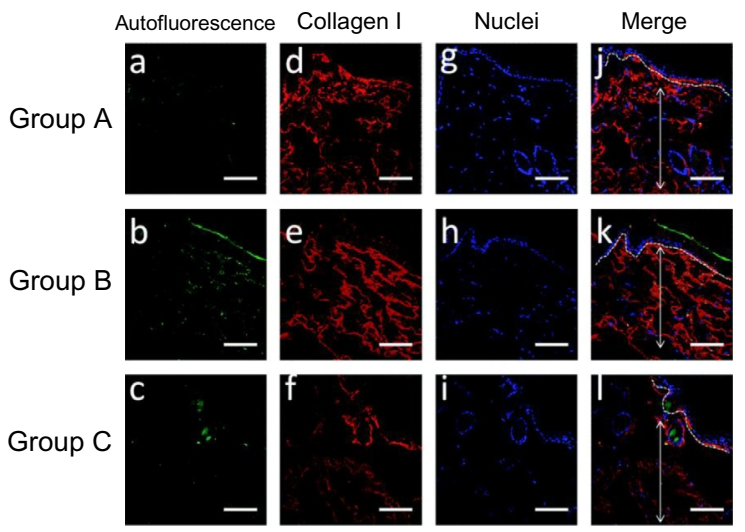

Fig. 8.19 Expression of

collagen on day 7. DAPI-labeled nuclei (blue) (d-f). Cy3conjugated secondary antibody (orange) (g-i). RhPDGF-BBeluting PLGA-collagen hybrid scaffold increases collagen I in the dermis (double arrow). Dashed lines indicate dermal-epidermal junction. Scale bar $=75 \mu \mathrm{m}$.

Autofluorescence $(\mathrm{a}-\mathrm{c})$. Reproduced with permission from Lee CH, Hsieh MJ, Chang SH, Lin YH, Liu SJ, Lin TY,

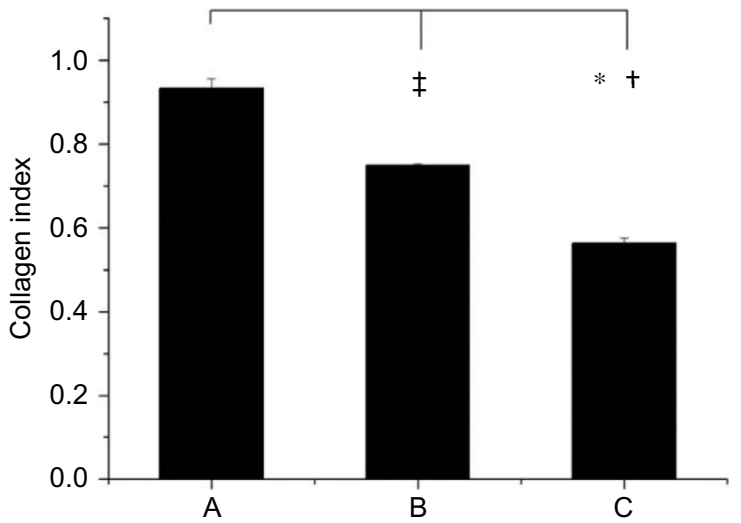
et al. Enhancement of diabetic wound repair using biodegradable nanofibrous metformin-eluting membranes: in vitro and in vivo. ACS Appl Mater Interfaces 2016;6:3979-86 with permission from The Royal Society of Chemistry.

creating a moist wound healing environment and oxygen exchanging in wound healing process. Also, the hydrogel exhibited a good antibacterial activity against $E$. coli bacteria indicating their possible application in wound dressing.

\subsection{Current challenges and future directions}

The future of wound dressing is a multifunctional device that could enhance the healing process, prevent infection, or effectively treat an infection when it occurs, with simultaneous monitoring of the wound status. To date, a few electrospun-based scaffolds are available in the market with intended application in tissue engineering from different companies including Ahlstrom Corporation (Finland), Espin Technologies (the United States), Hemcon Medical Technologies, Inc. (the United States), Nanofiber Solutions (the United States), and Neotherix (the United Kingdom). Although electrospinning is a well-established technology in wide range of applications, fabrication of ECM 
mimicking scaffolds are considered to be a primary challenge till now. A significant attention needs to be paid for creating ECM analog structures to promote better cellular penetration and scaffold remodeling since if cells cannot penetrate into nanofibrous structure, then it will be of little use especially in wound healing applications. As discussed earlier, electrospun fibrous mats offer all essential requirements for effective wound care; still, more significant insights are needed on some elementary aspects of fabrication process including various parameters, polymer selection, and active agents to be loaded to scale-up in cost-effective industrial scale and to possess excellent wound care properties.

\section{Acknowledgments}

B. B. acknowledges TUBITAK-BIDEB 2216, Research Fellowship Programme for Foreign Citizens for postdoctoral fellowship. A. S. acknowledges the Scientific and Technological Research Council of Turkey (TUBITAK), BIDEB 2221-Fellowships for Visiting Scientists, and Scientists on Sabbatical for the fellowship. T. U. acknowledges the Turkish Academy of Sciences-Outstanding Young Scientists Award Program (TUBA-GEBIP).

\section{References}

[1] Orsted H, Keast D, Forest-Lalande L, Mégie MF. Basic principles of wound healing. Wound Care Canada 2011;9:4-12.

[2] Barrientos S, Stojadinovic O, Golinko MS, Brem H, Tomic-Canic M. Growth factors and cytokines in wound healing. Wound Repair Regen 2008;16:585-601.

[3] Clark RAF. The molecular and cellular biology of wound repair. 2nd ed. New York: Plenum Press; 1995 [chapter 1].

[4] Gurtner GC, Werner S, Barrandon Y, Longaker MT. Wound repair and regeneration. Nature 2008;453:314-21.

[5] Harding KG, Morris HL, Patel GK. Science, medicine and the future: healing chronic wounds. Br Med J 2002;324:160-3.

[6] Percival NJ. Classification of wounds and their management. Surgery 2002;20:114-7.

[7] Sen CK, Gordillo GM, Roy S, Kirsner R, Lambert L, Hunt TK, et al. Human skin wounds: a major and snowballing threat to public health and the economy. Wound Repair Regen 2009; 17:763-71.

[8] Boateng JS, Matthews KH, Stevens HNE, Eccleston GM. Wound healing dressings and drug delivery systems: a review. J Pharm Sci 2008;97:2892-923.

[9] Eccleston GM. Wound dressings. In: Aulton ME, editor. Pharmaceutics: the science of dosage form design. New York: Churchill Livingstone; 2007. p. 264-71.

[10] Murphy PS, Evans GRD. Advances in wound healing: a review of current wound healing products. Plast Surg Int 2012;2012:190436.

[11] Kalashnikova I, Das S, Seal S. Nanomaterials for wound healing: scope and advancement. Nanomedicine 2015;10:2593-612.

[12] Agarwal S, Wendorff JH, Greiner A. Use of electrospinning technique for biomedical applications. Polymer 2008;49:5603-21. 
[13] Kanani AG, Bahrami SH. Review on electrospun nanofibers scaffold and biomedical applications. Trends Biomater Artif Organs 2010;24:93-115.

[14] Leung V, Ko F. Biomedical applications of nanofibers. Polym Adv Technol 2011;22:350-65.

[15] Senthamizhan A, Balusamy B, Uyar T. Glucose sensors based on electrospun nanofibers: a review. Anal Bioanal Chem 2016;408:1285-306.

[16] Thavasi V, Singh G, Ramakrishna S. Electrospun nanofibers in energy and environmental applications. Energy Environ Sci 2008;1:205-21.

[17] Abrigo M, McArthur SL, Kingshott P. Electrospun nanofibers as dressings for chronic wound care: advances, challenges, and future prospects. Macromol Biosci 2014;14:772-92.

[18] Chen H, Peng Y, Wu S, Tan LP. Electrospun 3D fibrous scaffolds for chronic wound repair. Materials 2016;9:272.

[19] Choi JS, Kim HS, Yoo HS. Electrospinning strategies of drug-incorporated nanofibrous mats for wound recovery. Drug Deliv Transl Res 2015;5:137-45.

[20] El-Sherbiny IM, Ali IH. Eco-friendly electrospun polymeric nanofibers-based nanocomposites for wound healing and tissue engineering. In: Thakur MK, editor. Eco-friendly polymer nanocomposites. India: Springer; 2015. p. 399-431.

[21] Hassiba AJ, Zowalaty MEE, Nasrallah GK, Webster TJ, Luyt AS, Abdullah AM, et al. Review of recent research on biomedical applications of electrospun polymer nanofibers for improved wound healing. Nanomedicine (Lond) 2016;11:715-37.

[22] Rieger KA, Birch NP, Schiffman JD. Designing electrospun nanofiber mats to promote wound healing - a review. J Mater Chem B 2013;1:4531-41.

[23] Stevens MM, George JH. Exploring and engineering the cell surface interface. Science 2005;310:1135-8.

[24] Manoukian OS, Ahmad A, Marin C, James R, Mazzocca AD, Kumbar SG. Bioactive nanofiber dressings for wound healing. In: Ågren M, editor. Wound healing biomaterials. Functional biomaterials, vol. 2. Elsevier, UK: Woodhead Publishing; 2016. p. 451-81.

[25] Mohiti-Asli M, Loboa EG. Nanofibrous smart bandages for wound care. In: Ågren M, editor. Wound healing biomaterials. Functional biomaterials, vol. 2. Elsevier, UK: Woodhead Publishing; 2016. p. 483-99.

[26] Norouzi M, Boroujeni SM, Omidvarkordshouli N, Soleimani Masoud. Advances in skin regeneration: application of electrospun scaffolds. Adv Healthcare Mater 2015; 4:1114-33.

[27] Mele E. Electrospinning of natural polymers for advanced wound care: towards responsive and adaptive dressings. J Mater Chem B 2016;4:4801-12.

[28] Powell HM, Supp DM, Boyce ST. Influence of electrospun collagen on wound contraction of engineered skin substitutes. Biomaterials 2008;29:834-43.

[29] Zhou T, Wang N, Xue Y, Ding T, Liu X, Mo X, et al. Electrospun tilapia collagen nanofibers accelerating wound healing via inducing keratinocytes proliferation and differentiation. Colloids Surf B 2016;143:415-22.

[30] Powell HM, Boyce ST. Fiber density of electrospun gelatin scaffolds regulates morphogenesis of dermal-epidermal skin substitutes. J Biomed Mater Res 2008;84A:1078-86.

[31] Dubský M, Kubinová Š, Širc J, Voska L, Zajícěk R, Zajícová A, et al. Nanofibers prepared by needleless electrospinning technology as scaffolds for wound healing. J Mater Sci Mater Med 2012;23:931-41.

[32] Uppal R, Ramaswamy GN, Arnold C, Goodband R, Wang Y. Hyaluronic acid nanofiber wound dressing - production, characterization, and in vivo behavior. J Biomed Mater Res Part B Appl Biomater 2011;97B:20-9. 
[33] Ji Y, Ghosh K, Li B, Sokolov JC, Clark RAF, Rafailovich MH. Dual-syringe reactive electrospinning of cross-linked hyaluronic acid hydrogel nanofibers for tissue engineering applications. Macromol Biosci 2006;6:811-7.

[34] Tchemtchoua VT, Atanasova G, Aqil A, Filée P, Garbacki N, Vanhooteghem O, et al. Development of a chitosan nanofibrillar scaffold for skin repair and regeneration. Biomacromolecules 2011;12:3194-204.

[35] Sheikh FA, Ju HW, Lee JM, Moon BM, Park HJ, Lee OJ, et al. 3D electrospun silk fibroin nanofibers for fabrication of artificial skin. Nanomedicine 2015;11:681-91.

[36] Ju HW, Lee OJ, Lee JM, Moon BM, Park HJ, Park YR, et al. Wound healing effect of electrospun silk fibroin nanomatrix in burn-model. Int J Biol Macromolec 2016;85:29-39.

[37] Li H, Wang M, Williams GR, Wu J, Sun X, Lv Y, et al. Electrospun gelatin nanofibers loaded with vitamins $\mathrm{A}$ and $\mathrm{E}$ as antibacterial wound dressing materials. RSC Adv 2016;6:50267-77.

[38] Rath G, Hussain T, Chauhan G, Garg T, Goyal AK. Collagen nanofiber containing silver nanoparticles for improved wound-healing applications. J Drug Target 2016;24:520-9.

[39] Jao WC, Yang MC, Lin CH, Hsu CC. Fabrication and characterization of electrospun silk fibroin/ $\mathrm{TiO}_{2}$ nanofibrous mats for wound dressings. Polym Adv Technol 2012;23:1066-76.

[40] Song DW, Kim SH, Kim HH, Lee KH, Ki CS, Park YH. Multi-biofunction of antimicrobial peptide-immobilized silk fibroin nanofiber membrane: implications for wound healing. Acta Biomater 2016;39:146-55.

[41] Murugan R, Ramakrishna S. Nano-featured scaffolds for tissue engineering: a review of spinning methodologies. Tissue Eng 2006;12:435-47.

[42] Zahedi P, Rezaeian I, Ranaei-Siadat SO, Jafari SH, Supaphol P. A review on wound dressings with an emphasis on electrospun nanofibrous polymeric bandages. Polym Adv Technol 2010;21:77-95.

[43] Bahrami H, Keshel SH, Chari AJ, Biazar E. Human unrestricted somatic stem cells loaded in nanofibrous PCL scaffold and their healing effect on skin defects. Artif Cells Nanomed Biotechnol 2016;44:1556-60.

[44] Augustine R, Dominic EA, Reju I, Kaimal B, Kalarikkal N, Thomas S. Electrospun polycaprolactone membranes incorporated with $\mathrm{ZnO}$ nanoparticles as skin substitutes with enhanced fibroblast proliferation and wound healing. RSC Adv 2014;4:24777-85.

[45] Pinzón-García AD, Cassini-Vieira P, Ribeiro CC, de Matos JCE, Barcelos LS, Cortes ME, et al. Efficient cutaneous wound healing using bixin-loaded PCL nanofibers in diabetic mice. J Biomed Mater Res B Appl Biomater 2016. http://dx.doi.org/10.1002/jbm.b.33724.

[46] Nguyen TTT, Ghosh C, Hwang SG, Tran LD, Park JS. Characteristics of curcumin-loaded poly(lactic acid) nanofibers for wound healing. J Mater Sci 2013;48:7125-33.

[47] Lee CH, Hsieh MJ, Chang SH, Lin YH, Liu SJ, Lin TY, et al. Enhancement of diabetic wound repair using biodegradable nanofibrous metformin-eluting membranes: in vitro and in vivo. ACS Appl Mater Interfaces 2014;6:3979-86.

[48] Said SS, El-Halfawy OM, El-Gowelli HM, Aloufy AK, Boraei NA, El-Khordagui LK. Bioburden-responsive antimicrobial PLGA ultrafine fibers for wound healing. Eur J Pharm Biopharm 2012;80:85-94.

[49] Khil MS, Cha DI, Kim HY, Kim IS, Bhattara N. Electrospun nanofibrous polyurethane membrane as wound dressing. J Biomed Mater Res Part B Appl Biomater 2003;67B:675-9.

[50] Unnithan AR, Pichiah PBT, Gnanasekaran G, Seenivasan K, Barakat NAM, Cha YS, et al. Emu oil-based electrospun nanofibrous scaffolds for wound skin tissue engineering. Colloids Surf A Physicochem Eng Asp 2012;415:454-60. 
[51] Sabitha M, Rajiv S. Preparation and characterization of ampicillin-incorporated electrospun polyurethane scaffolds for wound healing and infection control. Polym Eng Sci 2015;55:541-8.

[52] Chen JP, Chiang Y. Bioactive electrospun silver nanoparticles-containing polyurethane nanofibers as wound dressings. J Nanosci Nanotechnol 2010;10:7560-4.

[53] Lakshman LR, Shalumon KT, Nair SV, Jayakumar R, Nair SV. Preparation of silver nanoparticles incorporated electrospun polyurethane nano-fibrous mat for wound dressing. J Macromol Sci Pure Appl Chem 2010;47:1012-8.

[54] Nguyen TH, Kim YH, Song HY, Lee BT. Nano Ag loaded PVA nano-fibrous mats for skin applications. J Biomed Mater Res Part B Appl Biomater 2011;96B:225-33.

[55] Li Z, Liu X, Li Y, Lan X, Leung PH, Li J, et al. Composite membranes of recombinant silkworm antimicrobial peptide and poly (L-lactic acid) (PLLA) for biomedical application. Sci Rep 2016;6:31149.

[56] Ebrahimi-Hosseinzadeh B, Pedram M, Hatamian-Zarmi A, Salahshour-Kordestani S, Rasti M, Mokhtari-Hosseini ZB, et al. In vivo evaluation of gelatin/hyaluronic acid nanofiber as burn-wound healing and its comparison with ChitoHeal gel. Fibers Polym 2016;17:820-6.

[57] Sarhan WA, Azzazy HME, El-Sherbiny IM. Honey/chitosan nanofiber wound dressing enriched with Allium sativum and Cleome droserifolia: enhanced antimicrobial and wound healing activity. ACS Appl Mater Interfaces 2016;8:6379-90.

[58] Hajiali H, Summa M, Russo D, Armirotti A, Brunetti V, Bertorelli R, et al. Alginate-lavender nanofibers with antibacterial and anti-inflammatory activity to effectively promote burn healing. J Mater Chem B 2016;4:1686-95.

[59] Mohammadi MR, Rabbani S, Bahrami SH, Joghataei MT, Moayer F. Antibacterial performance and in vivo diabetic wound healing of curcumin loaded gum tragacanth/poly (ع-caprolactone) electrospun nanofibers. Mater Sci Eng C 2016;69:1183-91.

[60] Ganesh M, Aziz AS, Ubaidulla U, Hemalatha P, Saravanakumar A, Ravikumar R, et al. Sulfanilamide and silver nanoparticles-loaded polyvinyl alcohol-chitosan composite electrospun nanofibers: synthesis and evaluation on synergism in wound healing. J Ind Eng Chem 2016;39:127-35.

[61] Zhu L, Liu X, Du L, Jin Y. Preparation of asiaticoside-loaded coaxially electrospinning nanofibers and their effect on deep partial-thickness burn injury. Biomed Pharmacother 2016;83:33-40.

[62] Lee CH, Chao YK, Chang SH, Chen WJ, Hung KC, Liu SJ, et al. Nanofibrous rhPDGF-eluting PLGA-collagen hybrid scaffolds enhance healing of diabetic wounds. RSC Adv 2016;6:6276-84.

[63] Kim S, Park SG, Kang SW, Lee KJ. Nanofiber-based hydrocolloid from colloid electrospinning toward next generation wound dressing. Macromol Mater Eng 2016;301:818-26.

[64] Xu X, Zhou G, Li X, Zhuang X, Wang W, Cai Z, et al. Solution blowing of chitosan/PLA/ PEG hydrogel nanofibers for wound dressing. Fibers Polym 2016;17:205-11. 